\title{
Digital Correlation Microwave Polarimetry: Analysis and Demonstration
}

\author{
Jeffrey R. Piepmeier, Senior Member, IEEE, and Albin J. Gasiewski, Member, IEEE
}

\begin{abstract}
The design, analysis, and demonstration of a digital-correlation microwave polarimeter for use in Earth remote sensing is presented. We begin with an analysis of a three-level digital correlator and develop the correlator transfer function and radiometric sensitivity. A fifth-order polynomial regression is derived for inverting the digital correlation coefficient into the analog statistic. In addition, the effects of quantizer threshold asymmetry and hysteresis are discussed. A two-look unpolarized calibration scheme is developed for identifying correlation offsets. The developed theory and calibration method are verified using a 10.7 $\mathrm{GHz}$ and a $37.0 \mathrm{GHz}$ polarimeter. The polarimeters are based upon 1-GS/s three-level digital correlators and measure the first three Stokes parameters. Through experiment, the radiometric sensitivity is shown to approach the theoretical as derived earlier in the paper and the two-look unpolarized calibration method is successfully compared with results using a polarimetric scheme. Finally, sample data from an aircraft experiment demonstrates that the polarimeter is highly useful for ocean wind-vector measurement.
\end{abstract}

Index Terms-Correlator, digital, polarimetry, radiometer, Stokes vector.

\section{INTRODUCTION}

$\mathbf{M}$ ICROWAVE polarimeters are used to measure the polarization state or Stokes vector of microwave emission. Recent advances in the interpretation of polarimetric microwave thermal emission from the Earth's oceans and atmosphere have prompted the study of new retrieval techniques for near-surface ocean wind vectors (e.g., [1]) and mesospheric temperature profiles [2]. These techniques are facilitated by a more complete characterization of the polarization characteristics of the upwelling radiation field than obtainable using conventional single- or dual-polarization radiometers.

The Stokes vector is used to fully describe the second-order statistics of the quasimonochromatic radiation field at a point in space. The elements of the modified Stokes vector, in units of brightness temperature $(\mathrm{K})$, are directly related to the following ensemble averages of the incident transverse electric field components [3]:

$$
\bar{T}_{B}=\left[\begin{array}{c}
T_{v} \\
T_{h} \\
T_{U} \\
T_{V}
\end{array}\right]=\frac{\lambda^{2}}{\eta k}\left[\begin{array}{c}
\left\langle\left|E_{v}\right|^{2}\right\rangle \\
\left\langle\left|E_{h}\right|^{2}\right\rangle \\
2 \operatorname{Re}\left\langle E_{v} E_{h}^{*}\right\rangle \\
2 \operatorname{Im}\left\langle E_{v} E_{h}^{*}\right\rangle
\end{array}\right]
$$

Manuscript received September 28, 2000; revised September 4, 2001. This work was supported in part by NASA Grant NAGW-4191, ONR Grant N0014-95-1-0426, and ONR ASSERT Grant N0014-96-1-1007.

J. R. Piepmeier is with the NASA Goddard Space Flight Center, Greenbelt, MD 21146 USA (e-mail: jeff.piepmeier@gsfc.nasa.gov).

A. J. Gasiewski is with the NOAA Environmental Technology Laboratory, Boulder, CO 80305 USA.

Publisher Item Identifier S 0196-2892(00)10397-3. where $\lambda$ is the wavelength, $\eta$ is the impedance of the medium, and $k$ is Boltzmann's constant. Here, $E_{\alpha}$ is the phasor amplitude for polarization $\alpha(=v$ or $h)$ per unit solid angle and bandwidth. The first two parameters, $T_{v}$ and $T_{h}$, are the intensities in the vertical and horizontal polarizations and their sum is the total radiation intensity. The remaining two parameters contain information about the polarization characteristics of the radiation field. Specifically, $T_{U}$ indicates the degree and sense of linear polarization and $T_{V}$ of circular polarization. Partially polarized thermal radiation is specified by nonzero $T_{U}$ or $T_{V}$ and/or by $T_{v} \neq T_{h}$.

The parameters $T_{v}$ and $T_{h}$ can be measured using standard linearly polarized total power radiometers [4]. Detection of the third and fourth Stokes parameters, however, requires two additional measurements to effectively perform the correlations in (1). The various types of polarimetric radiometers fall into two basic categories: adding polarimeters (AP) and direct correlating polarimeters (DCP). The adding polarimeter uses measurements of the brightness temperature of at least two additional polarization states e.g., $45^{\circ}$ slant-linearly polarized $\left(T_{45^{\circ}}\right)$ and either left- or right-hand circularly polarized $\left(T_{l}\right.$ or $T_{r}$ ). From the four measured brightness temperatures and using the Stokes parameter rotational transformation [5], the third and fourth Stokes parameters can be determined, e.g.,

$$
\begin{aligned}
& T_{U}=T_{45^{\circ}}-T_{-45^{\circ}} \\
& T_{V}=T_{l}-T_{r} .
\end{aligned}
$$

The direct correlating polarimeter estimates $T_{U}$ and $T_{V}$ by cross-correlating the instantaneous voltage signals of the vertical and horizontal channels. The actual correlation can be performed by either analog or digital multiplying circuitry. If the time-varying voltages $v_{v}(t)$ and $v_{h}(t)$ are assumed to be stationary and ergodic [6], then the covariance estimate $\hat{R}_{v h}$ is

$$
\hat{R}_{v h}=\frac{1}{\tau} \int_{0}^{\tau} v_{v}(t) v_{h}(t) d t
$$

where $\tau$ is the integration time. Since the intermediate-frequency (IF) voltages are related to the incident field quantities by the receiving antenna's effective area and the receiver's signal transfer characteristics, measuring $R_{v h}$ is equivalent to measuring $T_{U}$

$$
\hat{T}_{U}=2 \hat{\rho} \sqrt{\hat{T}_{v, \mathrm{sys}} \hat{T}_{h, \mathrm{sys}}}
$$

where $\hat{\rho}=\hat{R}_{v h} / \hat{\sigma}_{v} \hat{\sigma}_{h}$ is the correlation coefficient, and $\hat{T}_{\alpha, \mathrm{sys}}$ are the system temperatures of the radiometers (assumed to be of the total power type) for polarizations $\alpha=v$ and $h$. A dualchannel superheterodyne receiver with a coherent local oscil- 
lator (LO) may be required to downconvert the radio frequency (RF) band of interest to accommodate the bandwidth and/or operating frequency of the analog multiplier or the digital correlator $\mathrm{A} / \mathrm{D}$ converters. If the signals are downconverted in-phase, $T_{U}$ is estimated. However, if the receiver is single-sideband and the signals are downconverted in phase-quadrature, then $T_{V}$ is estimated. Direct correlation without down conversion is also possible during undersampling or aliasing A/D converters [7].

Several mechanisms can contribute to calibration errors in (4) and (5). Antenna cross-polarization mixing and receiver imbalances can cause mixing of all four Stokes parameters, the amount of which must be known. One method for comprehensive calibration of the first three modified Stokes parameters uses a rotating polarized calibration standard [8]. The polarized standard presents to the receiver a strongly polarized but precisely determined radiation field and allows complete determination of the gains and offsets for the first three Stokes parameters. Calibration of the fourth Stokes parameter channel can be accomplished by insertion of an appropriate $90^{\circ}$ shift in the RF path using, e.g., a quarter wave plate [9]. Use of the polarized standard in space, however, requires additional hardware beyond the conventional ambient and cold blackbody standards that are commonly used.

In the implementation of (4) and (5) it is desirable to design a system that requires a minimal amount of calibration hardware. While an additive polarimeter can be used to determine $T_{U}$ or $T_{V}$, its response will generally require the in-situ identification of relatively large leakage gains $g_{U v}$ and $g_{U h}$ from $T_{v}$ and $T_{h}$

$$
v_{U}=g_{U v} T_{v}+g_{U h} T_{h}+g_{U U} T_{U}+g_{U V} T_{V}+o_{U}
$$

as well as the offset term $o_{U}$ [8]. While leakage gains can be minimized by proper tuning and balancing, elimination of long term drift in detection and video components of satellite instruments over months or years can be prohibitively expensive.

A solution to the aforementioned problem of precise measurement of either $T_{U}$ or $T_{V}$ can be found using digital correlation. Here, the RF (or IF) signals are sampled at the Nyquist rate, the digital samples cross-correlated using fast multiplication circuitry, and the correlation integral (4) performed via digital accumulation. Provided that the digitized signal contains no DC component and the A/D conversion is linear and unbiased, the correlation coefficient $\hat{\rho}$ can be obtained without leakage or offset. A further advantage of using a digital correlator with more than one bit (or two levels) of discretization is that in-situ calibration can be performed using only conventional ambient and cold unpolarized views, for example, an ambient blackbody target and cold space.

Digital correlation radiometry was first discussed by Weinreb [10] for use in autocorrelation spectrometers for radio astronomy. As shown by Weinreb in autocorrelation spectroscopy of Gaussian signals, only a single bit of quantization (i.e., two-level A/D conversion) is required to achieve $\sim 64 \%$ of the detection sensitivity of a perfect analog correlator system. As fast digital logic became more widely available the single-bit systems were replaced with three-level (reduced 2-bit or 1.6-bit) systems. The three-level correlator can obtain up to $81 \%$ of the detection sensitivity of the analog system [11].

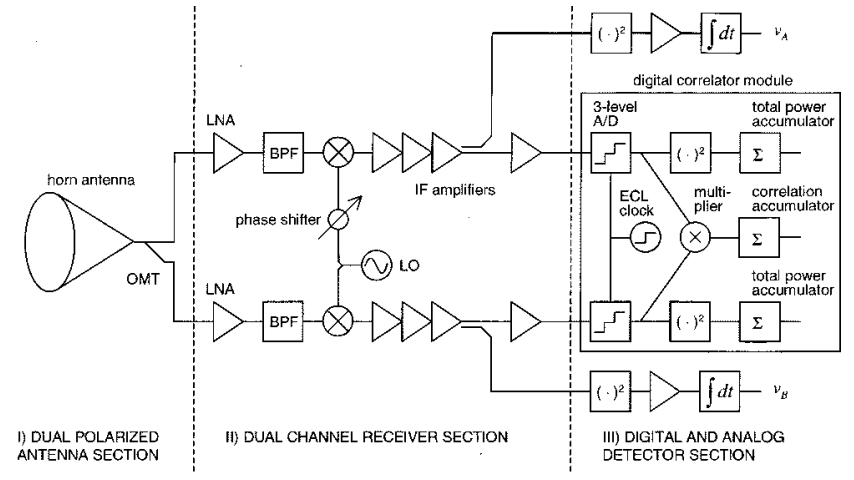

Fig. 1. Block diagram of a typical digital polarimetric radiometer. This direct correlating polarimeter, utilizes a dual polarized antenna, dual channel superheterodyne receiver and a three -level digital correlator. The IF signals are also coupled to traditional square law detectors and video amplifiers.

The availability of discrete high-speed digital logic has allowed spectrometers to operate over wider bandwidths, and both single-bit and two-bit correlators have now been implemented at clock-rates as high as $2 \mathrm{GS} / \mathrm{s}$ (e.g., [12]-[16]).

In this paper, we present the first demonstration of a polarimetric digital radiometer for earth remote sensing. Because of the relatively wide bandwidths required for earth remote sensing applications (typically tens to thousands of $\mathrm{MHz}$ ), the digital correlator has not been considered for use in space until recently. With the advent of high-speed radiation-hardened digital logic, bandwidths of hundreds of $\mathrm{MHz}$ have now become detectable using sensitive, short integration-time, digital correlating polarimeters. The block diagram of a generic digital correlating polarimeter is shown in Fig. 1. The major components are: i) a dual-polarized antenna, ii) a superheterodyne phase-coherent dual-channel receiver, and iii) a three-level digital cross-correlator. We begin in Section II with a description of digital correlation radiometry and discuss in Section III an investigation of systematic errors along with design implications. A novel two-look calibration of the correlation channel is discussed in Section IV. The results obtained from an airborne 1.6-bit X-band and $K_{a}$-band $1 \mathrm{GS} / \mathrm{s}$ digital polarimetric radiometer are reported in Section V, thus demonstrating the ability to properly calibrate and successfully operate a digital polarimetric radiometer.

\section{Digital Correlation Polarimeter}

The digital direct-correlating polarimeter has as its main distinguishing component a zero-lag digital cross-correlator. The digital correlator is composed of three sections: a pair of A/D converters, a digital multiplier, and accumulators. To fully understand the correlator operation, it is necessary to examine the relationship between the input signal statistics and the accumulator outputs. These relationships also provide a measure of the digital correlator sensitivity.

\section{A. Mean Statistics}

The input signals to a correlator $v_{a}(t)$ and $v_{b}(t)$ are modeled as jointly-Gaussian stationary random processes with root mean square (RMS) voltages $\sigma_{v_{a}}$ and $\sigma_{v_{b}}$ and correlation coefficient $\rho=\left(R_{v_{a} v_{b}}\right) /\left(\sigma_{v_{a}} \sigma_{v_{b}}\right)$. If the processes are sampled with period $T$ at or below their Nyquist rate, then the sample sequences 


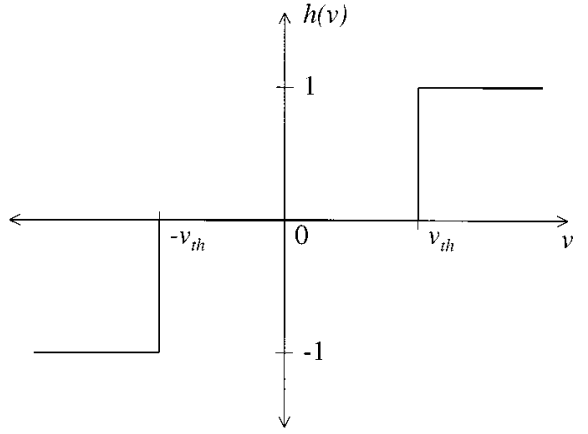

Fig. 2. Ideal transfer function of three-level A/D converter.

consist of independently and identically distributed pairs with the following joint Gaussian probability density function (pdf):

$$
\begin{aligned}
f\left(v_{a}, v_{b} ; \rho\right)= & \frac{1}{2 \pi \sigma_{v_{a}} \sigma_{v_{b}} \sqrt{1-\rho^{2}}} \\
& \times \exp \left[-\frac{\left(\frac{v_{a}}{\sigma_{v_{a}}}\right)^{2}-2 \rho \frac{v_{a} v_{b}}{\sigma_{v_{a}} \sigma_{v_{b}}}+\left(\frac{v_{b}}{\sigma_{v_{b}}}\right)^{2}}{2\left(1-\rho^{2}\right)}\right] .
\end{aligned}
$$

The three-level quantization performed on the input signals by the A/D converter can be modeled by a nonlinear transfer function

$$
h(v)= \begin{cases}1 & \text { if } v>v_{\mathrm{th}_{\alpha}} \\ -1 & \text { if } v<-v_{\mathrm{th}_{\alpha}} \\ 0 & \text { otherwise }\end{cases}
$$

where the quantities $\pm v_{t h_{\alpha}}$ are the threshold levels of the A/D converters (see also Fig. 2), with the subscript $\alpha$ denoting either channel $a$ or $b$. For typical CMOS or ECL circuits, $v_{\mathrm{th}_{\alpha}} \approx 0.05$ to $0.50 \mathrm{~V}$, therefore, the necessary microwave signal power can range from -12 to $+8 \mathrm{dBm}$ in a standard $50 \Omega$ system. DC blocking capacitors can be used prior to the A/D converters to make the input signals zero-mean as suggested by the model.

The outputs of the quantizers form a new pair of jointly-random processes denoted $h\left(v_{a}(n T)\right)$ and $h\left(v_{b}(n T)\right)$, where sample $n$ is taken at time $n T$. The second-order statistics of these sampled and quantized processes are the digital variances and covariance and are nonlinearly related to the first three Stokes parameters. For a measured sequence of $N$ samples, the estimated digital variances and covariance, denoted $\hat{s}_{\alpha}^{2}$ and $\hat{r}_{a b}$, are:

$$
\begin{aligned}
\hat{s}_{\alpha}^{2} & =\frac{1}{N} \sum_{n=1}^{N} h\left(v_{\alpha}(n T)\right)^{2} \\
\hat{r}_{a b} & =\frac{1}{N} \sum_{n=1}^{N} h\left(v_{a}(n T)\right) h\left(v_{b}(n T)\right) .
\end{aligned}
$$

\begin{tabular}{|c|c|c|c|c|c|c|c|c|c|}
\hline$h\left(v_{a}\right)$ & $h\left(v_{b}\right)$ & $\bar{A}^{+}$ & $A^{-}$ & $B^{+}$ & $B^{-}$ & $C_{A}$ & $\bar{C}_{B}$ & $C_{+}$ & $C_{-}$ \\
\hline 1 & 1 & 1 & 0 & 1 & 0 & 1 & 1 & 1 & 0 \\
\hline 1 & 0 & 1 & 0 & 0 & 0 & 1 & 0 & 0 & 0 \\
\hline 1 & -1 & 1 & 0 & 0 & 1 & 1 & 1 & 0 & 1 \\
\hline 0 & 1 & 0 & 0 & 1 & 0 & 0 & 1 & 0 & 0 \\
\hline 0 & 0 & 0 & 0 & 0 & 0 & 0 & 0 & 0 & 0 \\
\hline 0 & -1 & 0 & 0 & 0 & 1 & 0 & 1 & 0 & 0 \\
\hline-1 & 1 & 0 & 1 & 1 & 0 & 1 & 1 & 0 & 1 \\
\hline-1 & 0 & 0 & 1 & 0 & 0 & 1 & 0 & 0 & 0 \\
\hline-1 & -1 & 0 & 1 & 0 & 1 & 1 & 1 & 1 & 0 \\
\hline
\end{tabular}

These three statistical parameters are measured by accumulation of the outputs of a simple digital circuit such as shown in Fig. 3.

The statistics of $\hat{s}_{a}^{2}, \hat{s}_{b}^{2}$, and $\hat{r}_{a b}$ and their relationship to $T_{a}, T_{b}$, and $T_{U}$ are obtained by integrating the right-hand sides
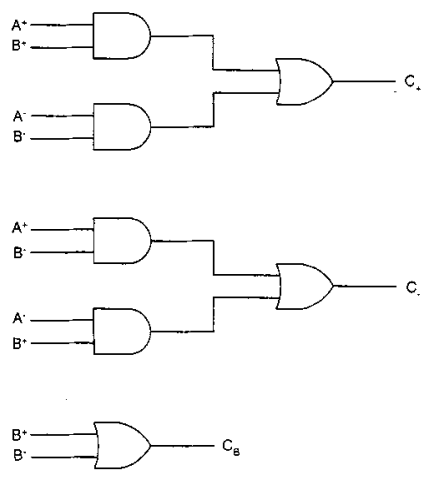

Fig. 3. Digital logic for the multiplier and squaring operations. The values of the boolean input variables $A^{+}, A^{-}, B^{+}$, and $B^{-}$are the outputs of the three-level A/D converters (see above table). The outputs $C_{A}$ and $C_{B}$ are the count-enable signals for the total-power accumulators and $C_{+}$and $C_{-}$are the count enable signals for the positive- and negative-correlation accumulators.

of (9) and (10) against the pdf (7). The expected value of the digital variance is

$$
\left\langle\hat{s}_{\alpha}^{2}\right\rangle=2\left[1-\Phi\left(\theta_{\alpha}\right)\right]
$$

where $\theta_{\alpha} \triangleq v_{\mathrm{th}_{\alpha}} / \sigma_{v_{\alpha}}$ and

$$
\Phi(x)=\frac{1}{\sqrt{2 \pi}} \int_{-\infty}^{x} e^{-t^{2} / 2} d t
$$

is the normal cumulative distribution function. Fig. 4 is a plot showing the relationship between the digital variance and root mean square (RMS) input voltage at a fixed threshold voltage. As will be shown in Section II-B, for maximum sensitivity in $T_{U}$, the value of $\theta_{\alpha}$ should be close to 0.61 . Inverting (11) yields a simple estimate for the signal standard deviation for a measured digital signal variance

$$
\hat{\theta}_{\alpha}=\Phi^{-1}\left(1-\frac{\left\langle\hat{s}_{\alpha}^{2}\right\rangle}{2}\right)
$$

or, in terms of antenna brightness temperatures

$$
\begin{aligned}
\hat{T}_{\mathrm{ANT}, \alpha} & =\frac{\hat{\sigma}_{v_{\alpha}}^{2} / R_{0}}{k B G_{\alpha}}-T_{\mathrm{REC}, \alpha} \\
& =\frac{v_{\mathrm{th}_{\alpha}}^{2}}{R_{0} k B G_{\alpha}}\left[\Phi^{-1}\left(1-\frac{\left\langle\hat{s}_{\alpha}^{2}\right\rangle}{2}\right)\right]^{-2}-T_{\mathrm{REC}, \alpha}
\end{aligned}
$$




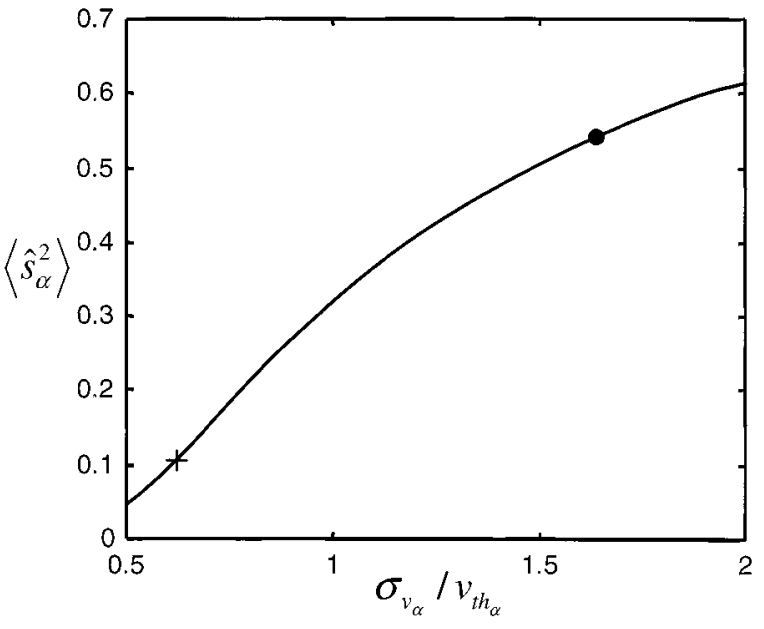

Fig. 4. Digital variance as a function of input RMS voltage at a fixed threshold level. The optimal threshold level for correlation $\theta_{\alpha}=0.61$ occurs at $\sigma_{v_{\alpha}} / v_{\mathrm{th}_{1_{\alpha}}}=1.64(\cdot)$ and for total power measurement. $\theta_{\alpha}=1.58$ occurs at $\sigma_{v_{\alpha}} / v_{\mathrm{tl}_{\alpha}}=0.633(+)$.

where $R_{0}$ is the system impedance, $B$ is the bandwidth, $G_{\alpha}$ is the system gain, and $T_{\mathrm{REC}, \alpha}$ is the receiver noise temperature. In general, the parameters $v_{\mathrm{th}_{\alpha}}^{2} /\left(R_{0} k B G_{\alpha}\right)$ and $T_{\mathrm{REC}, \alpha}$ are slowly time varying and represent system gains and offsets that must be identified via periodic calibration.

The relationship between the input correlation coefficient $\rho$ and the expected value of the digital covariance $r_{a b}$ is similarly straightforward and can be obtained by integrating the right-hand side of (10) against the joint pdf over two dimensions. The problem can be reduced, however, to an integration over one dimension using Price's theorem [17], [18]. Price's theorem relates the covariance of the input signals to the digital correlation coefficient

$$
\begin{aligned}
\frac{\partial r_{a b}}{\partial R_{v_{a} v_{b}}}= & \left\langle\frac{d h\left(v_{a}\right)}{d v_{a}} \frac{d h\left(v_{b}\right)}{d v_{b}}\right\rangle \\
= & \left\langle\left[\delta\left(v_{a}+v_{\mathrm{th}_{a}}\right)+\delta\left(v_{a}-v_{\mathrm{th}_{a}}\right)\right]\right. \\
& \left.\times\left[\delta\left(v_{b}+v_{\mathrm{th}_{b}}\right)+\delta\left(v_{b}-v_{\mathrm{th}_{b}}\right)\right]\right\rangle \\
= & f\left(v_{\mathrm{th}_{a}}, v_{\mathrm{th}_{b}} ; \rho\right)+f\left(v_{\mathrm{th}_{a}},-v_{\mathrm{th}_{b}} ; \rho\right) \\
& +f\left(-v_{\mathrm{th}_{a}}, v_{\mathrm{th}_{b}} ; \rho\right)+f\left(-v_{\mathrm{th}_{a}},-v_{\mathrm{th}_{b}} ; \rho\right) \\
= & f\left(\sigma_{v_{a}} \theta_{a}, \sigma_{v_{b}} \theta_{b} ; \rho\right)+f\left(-\sigma_{v_{a}} \theta_{a}, \sigma_{v_{b}} \theta_{b} ; \rho\right) \\
& +f\left(\sigma_{v_{a}} \theta_{a},-\sigma_{v_{b}} \theta_{b} ; \rho\right)+f\left(-\sigma_{v_{a}} \theta_{a},-\sigma_{v_{b}} \theta_{b} ; \rho\right)
\end{aligned}
$$

where $\delta$ is the Dirac impulse function. The input covariance can be related to the input correlation coefficient using the chain rule of differentiation

$$
\frac{\partial r_{a b}}{\partial \rho}=\frac{\partial r_{a b}}{\partial R_{v_{a} v_{b}}} \frac{\partial R_{v_{a} v_{b}}}{\partial \rho}=\sigma_{v_{a}} \sigma_{v_{b}} \frac{\partial r_{a b}}{\partial R_{v_{a} v_{b}}} .
$$

Thus, the digital correlation coefficient is a one-dimensional (1-D) integral of the pdf over $\rho$

$$
\begin{aligned}
r_{a b}= & \sigma_{v_{a}} \sigma_{v_{b}} \int_{0}^{\rho}\left[f\left(\sigma_{v_{a}} \theta_{a}, \sigma_{v_{b}} \theta_{b}, \rho^{\prime}\right)\right. \\
& +f\left(\sigma_{v_{a}} \theta_{a},-\sigma_{v_{b}} \theta_{b}, \rho^{\prime}\right)+f\left(-\sigma_{v_{a}} \theta_{a}, \sigma_{v_{b}} \theta_{b}, \rho^{\prime}\right) \\
& \left.+f\left(-\sigma_{v_{a}} \theta_{a},-\sigma_{v_{b}} \theta_{b}, \rho^{\prime}\right)\right] d \rho^{\prime} .
\end{aligned}
$$

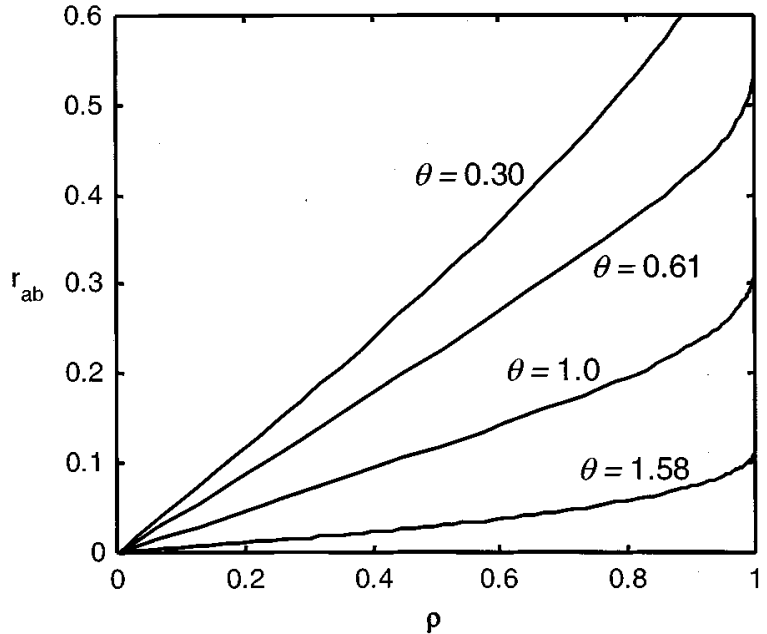

Fig. 5. The digital covariance $r_{a b}$ versus the input correlation coefficient $\rho$ for $\theta=0.30,0.61,1.0$, and 1.58

In practice, $\theta_{a}$ and $\theta_{b}$ are taken to be $\hat{\theta}_{a}$ and $\hat{\theta}_{b}$ from (13). The relationship between the input correlation coefficient and the digital covariance is plotted in Fig. 5 for fixed threshold levels $\theta_{\alpha}=0.30,0.61,1.0$, and 1.58 .

For a given $\hat{r}_{a b}$, the correlation estimate $\hat{\rho}$ is determined by nonlinear inversion of (17). The inversion technique must be carefully chosen so that systematic errors arising from the approximation are not larger than the statistical uncertainty of the estimate. This requirement is quite stringent. For example, from (5), a radiometer with a system temperature of $T_{\text {sys }}=500 \mathrm{~K}$ and a noise requirement of $\Delta T_{\mathrm{rms}}=0.1 \mathrm{~K}$ for the third or fourth Stokes parameter requires a measurement of $\rho$ with absolute error less than $0.1 \mathrm{~K} /(2 \cdot 500 \mathrm{~K})=1 \times 10^{-4}$. The two existing inversion techniques for three-level correlators are based upon power series inversions of either the bivariate normal integral [19] or the 1-D integral (17) [20]. In the former method [19] the inversion was derived for the cross-correlator, while for the latter method it was derived for the auto-correlator. Both share similar convergence characteristics, e.g., third-order expansions are required to obtain $0.1 \%$ accuracy or an absolute error of $10^{-4}$ for $|\rho| \lesssim 0.6$. The latter technique, however, is mathematically simpler and permits an analysis of the effects of system nonidealities (considered in Section III-A). Since this expression was originally derived for the autocorrelator, a new and more accurate expression tailored to the cross-correlator is presented here (the derivation is presented in Appendix I). First the integrand of (17) is approximated by a Taylor Series about $\rho^{\prime}=0$. Next, the series is integrated to obtain

$$
\begin{aligned}
r_{a b}= & \frac{2}{\pi} \exp \left[-\frac{1}{2}\left(\theta_{a}^{2}+\theta_{b}^{2}\right)\right] \\
& \times\left[\rho+\frac{1}{6}\left(\theta_{a}^{2}-1\right)\left(\theta_{b}^{2}-1\right) \rho^{3}+\frac{1}{120}\left(3-6 \theta_{a}^{2}+\theta_{a}^{4}\right)\right. \\
& \left.\times\left(3-6 \theta_{b}^{2}+\theta_{b}^{4}\right) \rho^{5}\right]+\mathcal{O}\left(\rho^{7}\right)
\end{aligned}
$$

Finally, a fifth-order power series inversion $[21,(3.6 .25)]$ is carried out on (18). The resulting estimate maps $\hat{r}_{a b}$ into $\hat{\rho}$ with ab- 
solute error of $\sim 10^{-5}$ for $|\rho| \lesssim 0.5$ and normalized threshold levels $\theta_{a}, \theta_{b}$ of $0.61 \pm 10 \%$

$$
\hat{\rho}=\frac{1}{c_{1}} \hat{r}_{a b}-\frac{c_{3}}{c_{1}^{4}} \hat{r}_{a b}^{3}+\left(3 \frac{c_{3}^{2}}{c_{1}^{7}}-\frac{c_{\tilde{s}}}{c_{1}^{6}}\right) \hat{r}_{a b}^{\tilde{b}}
$$

where

$$
\begin{aligned}
c_{1}= & \frac{2}{\pi} \exp \left[-\frac{1}{2}\left(\theta_{a}^{2}+\theta_{b}^{2}\right)\right] \\
c_{3}= & \frac{1}{3 \pi} \exp \left[-\frac{1}{2}\left(\theta_{a}^{2}+\theta_{b}^{2}\right)\right]\left(\theta_{a}^{2}-1\right)\left(\theta_{b}^{2}-1\right) \\
c_{5}= & \frac{1}{60 \pi} \exp \left[-\frac{1}{2}\left(\theta_{a}^{2}+\theta_{b}^{2}\right)\right]\left(3-6 \theta_{a}^{2}+\theta_{a}^{4}\right) \\
& \times\left(3-6 \theta_{b}^{2}+\theta_{b}^{4}\right) .
\end{aligned}
$$

Acceptable inversion errors for Earth science polarimetry are attainable using this fifth-order power series.

\section{B. Sensitivity}

A radiometer's fundamental sensitivity is limited by the available bandwidth, observation time, and receiver noise temperature. The radiometric sensitivity of a polarization correlating radiometer is

$$
\Delta T_{U, \mathrm{rms}}=\frac{\sigma_{\hat{\rho}}}{\partial \hat{\rho} / \partial \hat{T}_{U}}
$$

where $\sigma_{\hat{\rho}}$ is the standard deviation of the estimate $\hat{\rho}$. For sampled analog correlation using $N$-independent samples and small values of $\rho$, it can be shown that $\lim _{\rho \rightarrow 0} \sigma_{\hat{\rho}}=1 / \sqrt{N}$ [11]. Thus, using (5) and (21), the fundamental sensitivity for sampled correlation is

$$
\Delta T_{U, \mathrm{rms}}=\frac{2 \sqrt{T_{v, \mathrm{sys}} T_{h, \mathrm{sys}}}}{\sqrt{N}} .
$$

In continuous analog correlators, the number of independent Nyquist samples $N$ is equal to the product of twice-the-bandwidth (i.e., the Nyquist sampling rate) and the integration or accumulation time $\tau=N T: N=2 B \tau$. Thus, there is an equivalence between the sampled and continuous correlators

$$
\Delta T_{U, \mathrm{rms}}=\frac{2 \sqrt{T_{v, \mathrm{sys}} T_{h, \mathrm{sys}}}}{\sqrt{N}}=\sqrt{2} \frac{\sqrt{T_{v, \mathrm{sys}} T_{h, \mathrm{sys}}}}{\sqrt{B \tau}} .
$$

The above relationship is consistent with results previously derived for interferometers [22].

In the case of a digital correlator, the quantization noise of the A/D converter increases $\Delta T_{U, \text { rms }}$ above this value, the amount of which is a function of both the number of $\mathrm{A} / \mathrm{D}$ converter levels and the threshold voltages. Using a digital correlator, we have

$$
\Delta T_{U, \mathrm{rms}}=\frac{\sigma_{\hat{r}_{a b}}}{\partial \hat{r}_{a b} / \partial \hat{T}_{U}}
$$

For the three-level system with balanced channels $\left(\theta_{a}=\theta_{b}=\right.$ $\theta$ ), the sensitivity for vanishingly small correlation is (see Appendix II-A)

$$
\lim _{\rho \rightarrow 0} \Delta T_{U, \mathrm{rms}}=2 \pi[1-\Phi(\theta)] e^{\theta^{2}} \frac{\sqrt{T_{v, \mathrm{sys}} T_{h, \mathrm{sys}}}}{\sqrt{N}} .
$$

The impact of quantization noise can be minimized by proper selection of the threshold voltages $v_{t h_{\alpha}}$. The optimal value of $\theta$ (determined numerically) is 0.61 with a corresponding sensitivity of

$$
\Delta T_{U, \mathrm{rms}}=2.47 \frac{\sqrt{T_{v, \mathrm{sys}} T_{h, \mathrm{sys}}}}{\sqrt{N}} .
$$

Comparing this expression to (22), we find that the 1.6-bit digital correlator achieves $81 \%$ of the sensitivity available from an ideal analog correlator.

The total power channels are useful for normalized threshold level estimation as well as measurements of the first two Stokes parameters. The sensitivity of a total power channel can be calculated in a similar fashion by

$$
\Delta T_{\alpha, \mathrm{rms}}=\frac{\sigma_{\hat{s}_{\alpha}^{2}}}{\partial\left(\hat{s}_{\alpha}^{2}\right) / \partial \hat{T}_{\alpha}} .
$$

With the threshold levels $\theta_{a}=\theta_{b}=0.61$ (i.e., set for optimal cross-correlator sensitivity), the total power channels have a fundamental sensitivity of (see Appendix II-B)

$$
\Delta T_{\alpha, \mathrm{rms}}=2.20 \frac{T_{\mathrm{sys}, \alpha}}{\sqrt{N}} .
$$

The ideal (analog) total power radiometer has a sensitivity of $T_{\text {sys }} / \sqrt{N}$. Thus, a three-level digital total power radiometer can achieve $41 \%$ of the sensitivity of an ideal analog radiometer when the threshold voltages are optimized for maximum sensitivity in the cross-correlation channel. It is noted that in (28), the optimal sensitivity for the total power channels is not used because the threshold voltages were chosen to optimize the crosscorrelation channel. In other words, the threshold level value of 0.61 is the optimum value for small cross correlations but is not optimal for the total power channels. This choice is acceptable, however, because in a polarization correlating radiometer the total power channels are primarily used to measure the relative threshold level values. If the thresholds were to be set for optimum total-power sensitivity, the digital total-power radiometer could achieve $78 \%$ of the sensitivity of an analog radiometer with $\theta_{\alpha}=1.58$.

\section{SYSTEMATIC ERRORS}

Two sources of systematic errors in a polarimetric radiometer are analyzed in this section: 1) errors caused by threshold asymmetries in the A/D converters (sampler offsets) and 2) other correlator gain attenuating sources, including A/D hysteresis, timing skew, and phase differences. Design suggestions to mitigate each error are discussed.

\section{A. Sampler Offsets}

Threshold level asymmetries in the correlator A/D converters produce systematic errors in the variance and correlation measurements. When extreme accuracy is not required, the effects of these relatively small biases can be neglected. For high-accuracy applications, such as those found in microwave polarimetry for wind vector measurement, however, asymmetric threshold levels cause attenuation and offset variations that require compensation. We present here an analysis illustrating the second- 
order behavior of bias effects along with a simple analytical correction that can be incorporated into an in-situ radiometer calibration algorithm.

The three-level A/D converter with a transfer function given by (8) typically exhibits small DC offset voltages $v_{\delta_{\alpha}}$, which effectively cause the threshold levels to be asymmetric about ground potential. We define the normalized threshold asymmetry to be $\delta_{\alpha} \triangleq v_{\delta_{\alpha}} / \sigma_{v_{\alpha}}$. Incorporating this offset into the transfer function, we now have (see Fig. 6)

$$
h\left(v-v_{\delta_{\alpha}}\right)= \begin{cases}1 & \text { if } v>v_{\mathrm{th}_{\alpha}} \\ -1 & \text { if } v<-v_{\mathrm{th}_{\alpha}} . \\ 0 & \text { otherwise }\end{cases}
$$

Relations (9) and (10) can now be recomputed to reveal the effects of threshold offsets.

1) Correlation Channel: The digital correlation coefficient (17) including offsets becomes

$$
\begin{aligned}
r_{a b}= & \left.r_{a b}\right|_{\rho=0}+\sigma_{v_{a}} \sigma_{v_{b}} \\
& \times \int_{0}^{\rho}\left[f\left(\sigma_{v_{a}}\left(\theta_{a}+\delta_{a}\right), \sigma_{v_{b}}\left(\theta_{b}+\delta_{b}\right), \rho^{\prime}\right)\right. \\
& +f\left(\sigma_{v_{a}}\left(\theta_{a}+\delta_{a}\right), \sigma_{v_{b}}\left(-\theta_{b}+\delta_{b}\right), \rho^{\prime}\right) \\
& +f\left(\sigma_{v_{a}}\left(-\theta_{a}+\delta_{a}\right), \sigma_{v_{b}}\left(\theta_{b}+\delta_{b}\right), \rho^{\prime}\right) \\
& \left.+f\left(\sigma_{v_{a}}\left(-\theta_{a}+\delta_{a}\right), \sigma_{v_{b}}\left(-\theta_{b}+\delta_{b}\right), \rho^{\prime}\right)\right] d \rho^{\prime} .
\end{aligned}
$$

Equation (30) can be considered equivalent to (17) but with small gain and offset perturbations of order $\delta_{a}$ and $\delta_{b}$. We show here that the gain error is negligible if the input correlation coefficient is small. In contrast, the offset error is found to be an order of magnitude larger than the gain error. This correlation offset, however, is parameterized in terms of the threshold level offset and may be compensated via calibration using two unpolarized standards.

The correlator offset error arises from the constant of integration $\left.r_{a b}\right|_{\rho=0}$ in (30). This constant was not explicitly shown in (17) because ideally it is zero. The constant can be evaluated by taking the expected value of (10) with $\rho=0$ and using the modified definition of $h\left(v-v_{\delta_{\alpha}}\right)$ in (29):

$$
\left.r_{a b}\right|_{\rho=0}=\left.\left\langle h\left(v_{a}-v_{\delta_{a}}\right) h\left(v_{b}-v_{\delta_{b}}\right)\right\rangle\right|_{\rho=0}
$$

Clearly, when either threshold level is ideal (i.e., $\delta_{\alpha}=0$ ) the above term vanishes. A shift in both threshold levels, however, causes the offset error to become nonzero, the expected value of which can be separated into a product of two expected values since $v_{a}$ and $v_{b}$ are statistically independent when $\rho=0$. The resulting correlation offset is:

$$
\begin{aligned}
\left.r_{a b}\right|_{\rho=0}=[1-\Phi( & \left.\left.-\theta_{a}+\delta_{a}\right)-\Phi\left(\theta_{a}+\delta_{a}\right)\right] \\
& \times\left[1-\Phi\left(-\theta_{b}+\delta_{b}\right)-\Phi\left(\theta_{b}+\delta_{b}\right)\right]
\end{aligned}
$$

Assuming $\delta_{a}$ and $\delta_{b}$ are small allows (32) to be approximated using Taylor series expansions about $\pm \theta_{a}$ and $\pm \theta_{b}$. The first term in the product is

$$
\begin{aligned}
1- & \Phi\left(-\theta_{a}+\delta_{a}\right)-\Phi\left(\theta_{a}+\delta_{a}\right) \\
& =1-\left[\Phi\left(\theta_{a}\right)+\frac{1}{2} \sqrt{\frac{2}{\pi}} e^{-\frac{1}{2} \theta_{a}^{2}} \delta_{a}\right.
\end{aligned}
$$

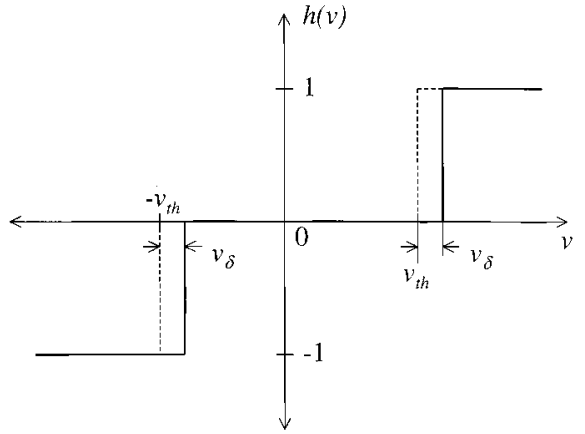

Fig. 6. Transfer function of three-level A/D converter with threshold offset $v_{\varepsilon}$.

$$
\begin{aligned}
- & \left.\frac{1}{4} \sqrt{\frac{2}{\pi}} e^{-\frac{1}{2} \theta_{a}^{2}} \theta_{a} \delta_{a}^{2}+\mathcal{O}\left(\delta_{a}^{3}\right)\right] \\
- & {\left[\Phi\left(-\theta_{a}\right)+\frac{1}{2} \sqrt{\frac{2}{\pi}} e^{-\frac{1}{2} \theta_{a}^{2}} \delta_{a}\right.} \\
& \left.+\frac{1}{4} \sqrt{\frac{2}{\pi}} e^{-\frac{1}{2} \theta_{a}^{2}} \theta_{a} \delta_{a}^{2}+\mathcal{O}\left(\delta_{a}^{3}\right)\right] \\
= & -\sqrt{\frac{2}{\pi}} e^{-\frac{1}{2} \theta_{a}^{2}} \delta_{a}+\mathcal{O}\left(\delta_{a}^{3}\right)
\end{aligned}
$$

where the $\delta_{a}^{2}$ terms cancel to leave an odd-valued function. The linear behavior of (33) makes the threshold asymmetry a significant source of error. The constant of integration in (30) is the product of two such terms:

$$
\begin{aligned}
\left.r_{a b}\right|_{\rho=0}= & {\left[-\sqrt{\frac{2}{\pi}} e^{-\frac{1}{2} \theta_{a}^{2}} \delta_{a}+\mathcal{O}\left(\delta_{a}^{3}\right)\right] } \\
& \times\left[-\sqrt{\frac{2}{\pi}} e^{-\frac{1}{2} \theta_{b}^{2}} \delta_{b}+\mathcal{O}\left(\delta_{b}^{3}\right)\right] \\
= & \frac{2}{\pi} \theta_{a} \theta_{b} \exp \left[-\frac{1}{2}\left(\theta_{a}^{2}+\theta_{b}^{2}\right)\right] \pi_{\delta}+\mathcal{O}\left(\delta_{a} \delta_{b}^{3}, \delta_{b} \delta_{a}^{3}\right)
\end{aligned}
$$

where

$$
\pi_{\delta} \triangleq \frac{\delta_{a}}{\theta_{a}} \frac{\delta_{b}}{\theta_{b}}
$$

is the normalized threshold offset product. The threshold asymmetries thus affect the digital correlation offset by an amount proportional to the normalized offset product. Expressed using voltages:

$$
\pi_{\delta}=\frac{v_{\delta_{a}} v_{\delta_{b}}}{v_{\mathrm{th}_{a}} v_{\mathrm{th}_{b}}}
$$

The above product is generally a slowly time varying hardware constant, but as will be shown in Section IV, it can be estimated using a conventional two-look unpolarized calibration.

The correlator gain perturbation is found by expanding the integrand of (30) in a three-dimensional (3-D) power series in $\rho^{\prime}, \delta_{a}$ and $\delta_{b}$, then integrating the resulting expansion with respect to $\rho^{\prime}$. The algebra involved (see Appendix III) is cumbersome, although the result can be expressed as a sum of two 
series. The first series $r_{a b} \mid \delta_{a}=\delta_{b}=0$ is the ideal relationship between $\rho$ and $r$ given by (17). The second series is an error series $\delta r_{a b}\left(\delta_{a}, \delta_{b}, \rho\right)$ caused by nonzero threshold offsets $\delta_{a}$ and $\delta_{b}$. Collecting these terms we have:

$$
\begin{aligned}
r_{a b}= & \left.r_{a b}\right|_{\rho=0}+\left.r_{a b}\right|_{\delta_{a}=\delta_{b}=0}+\frac{2}{\pi} \exp \left[-\frac{1}{2}\left(\theta_{a}^{2}+\theta_{b}^{2}\right)\right] \\
& \times\left[-\frac{1}{2}\left[\left(1-\theta_{a}^{2}\right) \delta_{a}^{2}+\left(1-\theta_{b}^{2}\right) \delta_{b}^{2}\right] \rho\right. \\
& +\frac{1}{4} \delta_{a} \delta_{b}\left(1-2 \theta_{a}^{2}\right)\left(1-2 \theta_{b}^{2}\right) \rho^{2} \\
& -\frac{1}{6}\left[\left(6 \theta_{a}^{2}+\theta_{a}^{4}+3\right)\left(1-\theta_{b}^{2}\right) \delta_{a}^{2}+\left(1-\theta_{a}^{2}\right)\right. \\
& \left.\left.\times\left(6 \theta_{b}^{2}+\theta_{b}^{4}+3\right) \delta_{b}^{2}\right] \rho^{3}\right]+\mathcal{O}\left(\rho^{4}, \delta^{3}\right)
\end{aligned}
$$

The above series is truncated at $\mathcal{O}\left(\rho^{4}\right)$ and $\mathcal{O}\left(\delta^{3}\right)$. Assuming that the nominal threshold levels are equal to the optimal value $\left(\theta_{\alpha}=0.61\right)$ the error series becomes:

$$
\begin{array}{r}
\delta r_{a b}\left(\delta_{a}, \delta_{b}, \rho\right) \approx-0.3140\left(\delta_{a}^{2}+\delta_{b}^{2}\right) \rho+0.0164 \delta_{a} \delta_{b} \rho^{2} \\
-0.5621\left(\delta_{a}^{2}+\delta_{b}^{2}\right) \rho^{3}
\end{array}
$$

The error series is a sum of components that are $\mathcal{O}\left(\delta^{2} \rho\right), \mathcal{O}\left(\delta^{2} \rho^{2}\right)$, and $\mathcal{O}\left(\delta^{2} \rho^{3}\right)$, respectively.

To determine which components of the series are significant we assume that $\rho=0.1$ and $\delta_{\alpha}=\delta_{\beta}=\delta$ for which the absolute values of the three components are

$$
\begin{aligned}
\left|-0.3140\left(\delta_{a}^{2}+\delta_{b}^{2}\right) \rho\right| & \approx 0.044 \delta^{2} \\
\left|0.0164 \delta_{a} \delta_{b} \rho^{2}\right| & \approx 0.00016 \delta^{2} \\
\left|-0.5621\left(\delta_{a}^{2}+\delta_{b}^{2}\right) \rho^{3}\right| & \approx 0.00079 \delta^{2}
\end{aligned}
$$

To render these error terms insignificant, the quantity $\delta^{2}$ must be sufficiently small. Using the previous criterion that all errors in $\rho$ of magnitude $\lesssim 10^{-5}$ are negligible, the normalized threshold offsets should be no larger than $10^{-2}$, that is, $v_{\delta_{\alpha}} \leq 10^{-2} \sigma_{v_{\alpha}}$. This is readily attainable using precision electronics for $\sigma_{v_{\alpha}} \sim$ $0.5 \mathrm{~V}$. If threshold offsets are not small enough, then the offsets should at least be controlled to render insignificant the higherorder terms (e.g., $\rho^{2}, \rho^{3} \ldots$ ), in which case only a correctable gain error occurs. For this latter case, it is sufficient that $v_{\delta_{\alpha}} \leq$ $10^{-1} \sigma_{v_{\alpha}}$ to cause the magnitude of the $\rho^{2}$ and $\rho^{3}$ terms to be less than $10^{-5}$. The remaining error is linear in $\rho$ and can be modeled as an effective change in the correlator gain:

$$
\begin{aligned}
r_{a b}= & \left.r_{a b}\right|_{\rho=0}+\frac{2}{\pi} \exp \left[-\frac{1}{2}\left(\theta_{a}^{2}+\theta_{b}^{2}\right)\right] \\
& \times\left(1-\frac{1}{2}\left[\left(1-\theta_{a}^{2}\right) \delta_{a}^{2}+\left(1-\theta_{b}^{2}\right) \delta_{b}^{2}\right]\right) \rho+\mathcal{O}\left(\rho^{2}, \delta_{b}^{3}\right)
\end{aligned}
$$

Note that the ideal correlator output $\left.r_{a b}\right|_{\delta_{a}=\delta_{b}=0}$ is implicitly included in (40) through terms linear in $\rho$. Typically the threshold offsets are small enough so that the gain perturbation is a only few percent.

2) Total Power Channel: The effect of threshold asymmetry on the total power channels is a perturbed system gain and offset along with a residual nonlinearity that we show to be negligible. Consider the expected value of the total power output

$$
\left\langle\hat{s}_{\alpha}^{2}\right\rangle=\Phi\left(-\theta_{\alpha}+\delta_{\alpha}\right)+1-\Phi\left(\theta_{\alpha}+\delta_{\alpha}\right)
$$

This expression is a simple extension of (11) but includes the threshold asymmetry. The above can be approximated in $\delta_{\alpha}$ as

$$
\left\langle\hat{s}_{\alpha}^{2}\right\rangle=\Phi\left(-\theta_{\alpha}\right)+1-\Phi\left(\theta_{\alpha}\right)+\frac{1}{2} \sqrt{\frac{2}{\pi}} e^{-\theta_{\alpha}^{2} / 2} \theta_{\alpha} \delta_{\alpha}^{2}+\mathcal{O}\left(\delta_{\alpha}^{4}\right)
$$

Similar to the correlation channel, the expected value of the total power channel is a sum of the ideal output and an additional error series. We can now show that part of the error series can be combined with the ideal output to compute a modified system gain and offset, with the residual (nonlinear) component being insignificant. If all functions of $\theta_{\alpha}$ are approximated by a power series expansion

$$
\begin{aligned}
\Phi\left(-\theta_{\alpha}\right)+1-\Phi\left(\theta_{\alpha}\right) & =1-\sqrt{\frac{2}{\pi}} \theta_{\alpha}+\frac{1}{6} \sqrt{\frac{2}{\pi}} \theta_{\alpha}^{3}+\mathcal{O}\left(\theta_{\alpha}^{5}\right) \\
e^{-\theta_{\alpha}^{2} / 2} & =1-\frac{1}{2} \theta_{\alpha}^{2}+\mathcal{O}\left(\theta_{\alpha}^{4}\right)
\end{aligned}
$$

then (41) can be written

$$
\begin{aligned}
\left\langle\hat{s}_{\alpha}^{2}\right\rangle= & {\left[1-\sqrt{\frac{2}{\pi}} \theta_{\alpha}+\frac{1}{6} \sqrt{\frac{2}{\pi}} \theta_{\alpha}^{3}+\mathcal{O}\left(\theta_{\alpha}^{5}\right)\right] } \\
& +\frac{1}{2} \sqrt{\frac{2}{\pi}}\left[\theta_{\alpha}-\frac{1}{2} \theta_{\alpha}^{3}+\mathcal{O}\left(\theta_{\alpha}^{5}\right)\right] \delta_{\alpha}^{2}+\mathcal{O}\left(\delta_{\alpha}^{4}\right) \\
= & -\sqrt{\frac{2}{\pi}}\left(1-\frac{1}{2} \delta_{\alpha}^{2}\right) \theta_{\alpha} \\
& +\frac{1}{6} \sqrt{\frac{2}{\pi}}\left(1-\frac{3}{2} \delta_{\alpha}^{2}\right) \theta_{\alpha}^{3}+\mathcal{O}\left(\theta_{\alpha}^{5}, \delta_{\alpha}^{4}\right) \\
= & \left(1-\frac{1}{2} \delta_{\alpha}^{2}\right)\left[1-\sqrt{\frac{2}{\pi}} \theta_{\alpha}+\frac{1}{6} \sqrt{\frac{2}{\pi}} \theta_{\alpha}^{3}+\mathcal{O}\left(\theta_{\alpha}^{5}\right)\right] \\
& +\frac{1}{2} \delta_{\alpha}^{2}-\frac{1}{6} \sqrt{\frac{2}{\pi}} \delta_{\alpha}^{2} \theta_{\alpha}^{3}+\mathcal{O}\left(\delta_{\alpha}^{4}\right) \\
= & \left(1-\frac{1}{2} \delta_{\alpha}^{2}\right)\left\langle\hat{s}_{\alpha}^{2}\right\rangle_{\delta_{\alpha}}=0+\frac{1}{2} \delta_{\alpha}^{2} \\
& -\frac{1}{6} \sqrt{\frac{2}{\pi}} \delta_{\alpha}^{2} \theta_{\alpha}^{3}+\mathcal{O}\left(\delta_{\alpha}^{4}\right)
\end{aligned}
$$

There is a gain term affecting the total power channel output by a factor of $\left(1-(1 / 2) \delta_{\alpha}^{2}\right)$ and an offset of approximately $(1 / 2) \delta_{\alpha}^{2}$. This additional system gain and offset is easily identified via a standard two-look calibration. The nonlinear residual is

$$
-\frac{1}{6} \sqrt{\frac{2}{\pi}} \delta_{\alpha}^{2} \theta_{\alpha}^{3}+\mathcal{O}\left(\delta_{\alpha}^{4}\right)
$$

Assuming the optimal value for the threshold levels $\left(\theta_{\alpha}=0.61\right)$, the above residual is found to be $\sim 10^{-2} \delta_{\alpha}^{2}$. If $\delta_{\alpha} \lesssim 10^{-2}$, then the nonlinear residual term becomes $\sim 10^{-6}$, which is insignificant for either total power or threshold estimation. 


\section{B. Other Correlator Gain Attenuating Sources}

Analog-to-digital converter hysteresis acts to reduce the correlation output by an amount proportional to the magnitude of the hysteresis. This effect has been modeled by D'Addario, et al. [19] assuming a uniformly distributed region of uncertainty about the nominal threshold. However, this statistical model underestimates the attenuation effect because the hysteresis is treated as a process that is statistically independent from the signal. Rather, hysteresis is a nonstationary process in which the current threshold level is dependent upon the previous value of the input signal. To make a more accurate assessment of hysteresis a Monte-Carlo simulator was constructed to demonstrate the effect on the gain of the correlation channel. The simulator is based upon an A/D converter transfer function of the form: (Please see the equation at the bottom of the page.) where $v_{\mathrm{hys}, \alpha}$ is the hysteresis voltage. The transfer function is graphically illustrated in Fig. 7. Input correlation coefficients in the range $-0.1<\rho<0.1$ were tested with varying levels of hysteresis using $2^{14}$ Monte-Carlo samples for each case. In Fig. 8, the correlator output $r_{a b}$ is plotted for values of hysteresis in the range $0 \leq v_{\text {hys }, \alpha} / \sigma_{v_{\alpha}} \leq 0.333$. The computed relative attenuation on $r_{a b}$ due to the hysteresis is practically independent of $\rho$ over this range. The results of the statistical model of [19] for the same conditions are also plotted. Compared to the Monte-Carlo simulations, the statistical model underestimates the attenuation effect by a factor of $\sim 10$ at $v_{\text {hys }, \alpha} / \sigma_{v_{\alpha}}=0.1$.

The results suggest that hysteresis has a moderate effect on the correlator output. A reduction in correlator gain of $1 \%$ is caused by a hysteresis voltage equal to $2 \%$ of the RMS signal voltage. For a $0 \mathrm{dBm}$ signal into $50 \Omega$ this reduction would be caused by a hysteresis voltage of only $4.4 \mathrm{mV}$. Appropriate care should be taken in the design of the A/D converter circuitry. Alternatively, in-situ correction could be made using a polarimetric calibration system or with precise knowledge of the hysteresis levels.

Timing skew between the A/D converters or (equivalently) additional delay in one of the RF or IF paths has a similar effect of reducing the correlator output. The baseband signals at the correlator input can be modeled by

$$
\begin{aligned}
& v_{a}(t)=v_{a}^{0}(t)+v_{c}(t) \\
& v_{b}(t)=v_{b}^{0}(t-\Delta t)+v_{c}(t-\Delta t)
\end{aligned}
$$

where $v_{a}^{0}(t), v_{b}^{0}(t)$, and $v_{c}(t)$ are mutually uncorrelated and wide-sense stationary, and $\Delta t$ is an additional path delay or timing skew. If $v_{c}(t)$ is bandlimited then the cross-correlation function is:

$$
R_{v_{a} v_{b}}(\Delta t)=\rho \sigma_{v_{a}} \sigma_{v_{b}} \operatorname{sinc}(2 \pi B \Delta t)
$$

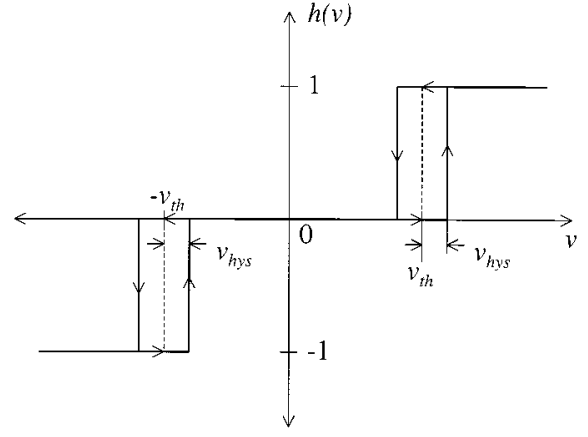

Fig. 7. Transfer function of three-level A/D converter with hysteresis magnitude $v_{\text {lyss }}$.

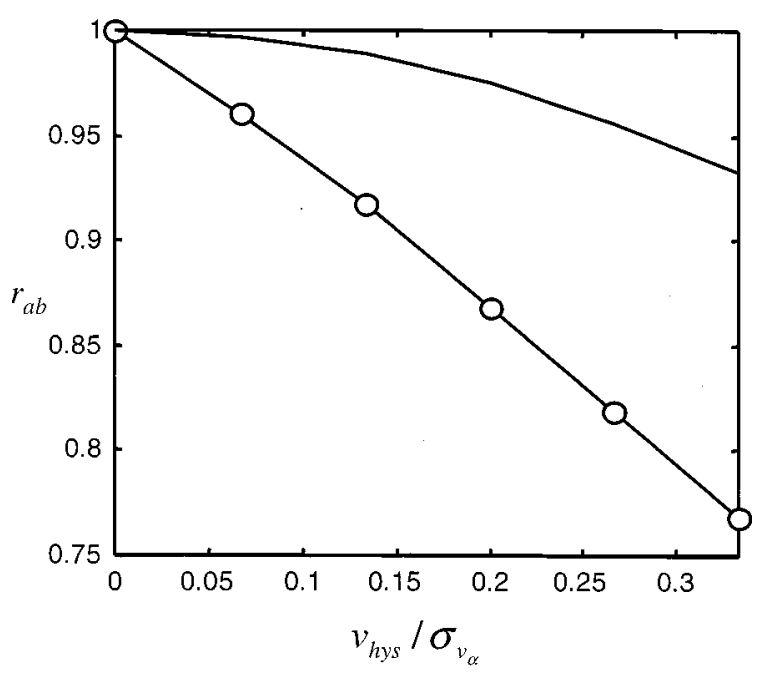

Fig. 8. The reduction in the digital correlator output as a function of hysteresis amplitude. The results of the Monte-Carlo model (solid line with open circles) shows that the statistical model [18] (solid line) significantly under estimates the effect.

where $B$ is the bandwidth or bandlimiting cutoff frequency of $v_{c}(t)$, and the function $\operatorname{sinc}(x) \triangleq(\sin x) /(x)$. Thus, timing skew will reduce the measured correlation coefficient. For example, a $1 \%$ reduction would be caused by $\Delta t=0.039 B^{-1}$. For a $500 \mathrm{MHz}$ bandwidth, this corresponds to $\Delta t=78 \mathrm{ps}$ or $\sim 24 \mathrm{~mm}$ of free-space IF path delay.

Phase differences between the two signals will also cause an attenuation of the measured correlation coefficient. Phase differences can be caused by LO distribution phase errors and phase imbalances between amplifiers, filters, waveguides, and other transmission paths of the respective signals. If the phase error is

$$
h\left(v\left(n T ; v_{\mathrm{hys}, \alpha}\right)=\left\{\begin{array}{llll}
1 & \text { if } v(n T)>v_{\mathrm{th}_{\alpha}}+v_{\mathrm{hys}, \alpha} & \text { AND } \quad h[v((n-1) T)] \neq 1 \\
1 & \text { if } v(n T)>v_{\mathrm{th}_{\alpha}}-v_{\mathrm{hys}, \alpha} & \text { AND } h[v((n-1) T)]=1 \\
-1 & \text { if } v(n T)<-v_{\mathrm{th}_{\alpha}}+v_{\mathrm{hys}, \alpha} & \text { AND } \quad h[v((n-1) T)]=-1 \\
-1 & \text { if } v(n T)<-v_{\mathrm{th}_{\alpha}}-v_{\mathrm{hys}, \alpha} & \text { AND } & h[v((n-1) T)] \neq-1 \\
0 & \text { otherwise. }
\end{array}\right.\right.
$$


denoted $\Delta \gamma$, then the cross-correlation at the correlator inputs is

$$
R_{v_{a} v_{b}}=\rho \sigma_{v_{a}} \sigma_{v_{b}} \cos (\Delta \gamma)
$$

For example, a $10^{\circ}$ or $20^{\circ}$ phase difference will cause a $1.5 \%$ or $6 \%$ reduction in the correlation coefficient, respectively.

\section{CALIBRATiOn}

Calibration of a digital polarimeter entails the periodic identification of slowly time-varying system hardware parameters. For the total power channels, these constants are the system gain and offset. For the polarization correlating channel, the threshold-offset product (37) in Section III-A1 as well as any other additive offsets (such as those originating from correlated LO noise) must be identified. As shown below, these parameters can be estimated using the simple hot and cold views of unpolarized blackbody standards as obtained during conventional total power channel calibration.

\section{A. Total Power Channel Calibration}

Identification of the gain and offset of the total power channels in (14) allow an antenna temperature estimate to be made. The output of the total power channel is related to the antenna temperature estimate by:

$$
\left[\Phi^{-1}\left(1-\frac{\hat{s}_{\alpha}^{2}}{2}\right)\right]^{-2}=g_{\alpha}\left(\hat{T}_{\mathrm{ANT}, \alpha}+T_{\mathrm{REC}, \alpha}\right)
$$

where the left hand side is the linearized digital variance, $g_{\alpha}$ is the radiometer system gain in $\mathrm{K}^{-1}$, and the receiver temperature $T_{\mathrm{REC}, \alpha}$ is the system offset.

The gain and offset can be estimated if the radiometer is presented two known antenna temperatures of differing values. The digital variance measurements corresponding to the hot and cold calibration temperatures, denoted $T_{\mathrm{CAL}}^{\text {hot }}$ and $T_{\mathrm{CAL}}^{\text {cold }}$, are:

$$
\begin{aligned}
& {\left[\Phi^{-1}\left(1-\frac{\hat{s}_{\alpha}^{2 \text { hot }}}{2}\right)\right]^{-2}=\hat{g}_{\alpha}\left(T_{\mathrm{CAL}}^{\text {hot }}+\hat{T}_{\mathrm{REC}, \alpha}\right)} \\
& {\left[\Phi^{-1}\left(1-\frac{\hat{s}_{\alpha}^{2 \text { cold }}}{2}\right)\right]^{-2}=\hat{g}_{\alpha}\left(T_{\mathrm{CAL}}^{\text {cold }}+\hat{T}_{\mathrm{REC}, \alpha}\right)}
\end{aligned}
$$

This system is easily solved for $\hat{g}_{\alpha}$ and $\hat{T}_{\mathrm{REC}, \alpha}$, see the equation at the bottom of the page. With the two system parameters properly identified, the estimated antenna temperature as measured by the total power radiometer is

$$
\hat{T}_{\mathrm{ANT}, \alpha}=\frac{1}{\hat{g}_{\alpha}}\left[\Phi^{-1}\left(1-\frac{\hat{s}_{\alpha}^{2}}{2}\right)\right]^{-2}-\hat{T}_{\mathrm{REC}, \alpha}
$$

\section{B. Correlator Channel Calibration}

Estimation of the digital correlator system parameters can be achieved using the same hot and cold calibration looks required for the calibration of the total-power channels. There are two unknown calibration offsets. The first is the correlation bias $\rho_{0}$, caused by correlated LO thermal noise. The second is the digital correlation offset $\left.r_{a b}\right|_{\rho=0}$, which is caused by threshold level asymmetry and is subtracted from the digital correlator output prior to conversion into the continuous correlation coefficient. The expected value of the correlator output given an unpolarized brightness field at the antenna input (i.e., $T_{U}=0$ ) is

$$
\left.r_{a b}\right|_{T_{U}=0}=c_{0} \pi_{\delta}+c_{1} \rho_{0}+c_{3} \rho_{0}^{3}+c_{5} \rho_{0}^{5}
$$

where

$$
c_{0}=\frac{2}{\pi} \theta_{a} \theta_{b} \exp \left[-\frac{1}{2}\left(\theta_{a}^{2}+\theta_{b}^{2}\right)\right]
$$

$c_{1}, c_{3}$, and $c_{5}$ are given by (20), and $\pi_{\delta}$ is the offset product (37). The fifth-order term $c_{5} \rho_{0}^{5}$ can be ignored if $\rho_{0}<0.1$, which is the case when $T_{U}$ is equal or close to zero.

The two calibration targets provide unpolarized emission at two different radiation intensities. Sequential views of the hot and cold targets provide the digital correlation measurements $\hat{r}_{a b}^{\text {hot }}$ and $\hat{r}_{a b}^{\text {cold }}$ for the hot and cold looks, respectively. Using these two measurements a system of equations can be formed:

$$
\begin{aligned}
\hat{r}_{a b}^{\text {hot }} & =c_{0}^{\text {hot }} \hat{\pi}_{\delta}+c_{1}^{\text {hot }} \hat{\rho}_{0}+c_{3}^{\text {hot }} \hat{\rho}_{0}^{3} \\
\hat{r}_{a b}^{\text {cold }} & =c_{0}^{\text {cold }} \hat{\pi}_{\delta}+c_{1}^{\text {cold }} \hat{\rho}_{0}+c_{3}^{\text {cold }} \hat{\rho}_{0}^{3}
\end{aligned}
$$

The coefficients $c_{i}^{\text {hot }}$ and $c_{i}^{\text {cold }}$ are computed by using the relative threshold values $\theta_{\alpha}^{\text {hot }}$ and $\theta_{\alpha}^{\text {cold }}$, respectively. Using only a third-order expansion in $\rho_{0}$ allows the above system to be solved analytically. An estimate of the threshold-offset product can be found by:

$$
\hat{\pi}_{\delta}=\frac{\hat{r}_{a b}^{\text {cold }}-c_{1}^{\text {cold }} \hat{\rho}_{0}-c_{3}^{\text {cold }} \hat{\rho}_{0}^{3}}{c_{0}^{\text {cold }}}
$$

$$
\begin{aligned}
& g_{\alpha}=\frac{\left[\Phi^{-1}\left(1-\frac{\hat{s}_{\alpha}^{2 \text { lot }}}{2}\right)\right]^{-2}-\left[\Phi^{-1}\left(1-\frac{\hat{s}_{\alpha}^{2 \text { cold }}}{2}\right)\right]^{-2}}{T_{\mathrm{CAL}}^{\text {hot }}-T_{\mathrm{CAL}}^{\text {cold }}} \\
& \hat{T}_{\mathrm{REC}, \alpha}=\frac{T_{\mathrm{CAL}}^{\mathrm{hot}}\left[\Phi^{-1}\left(1-\frac{\hat{s}_{\alpha}^{2 \text { cold }}}{2}\right)\right]^{-2}-T_{\mathrm{CAL}}^{\text {cold }}\left[\Phi^{-1}\left(1-\frac{\hat{s}_{\alpha}^{2 \text { loot }}}{2}\right)\right]^{-2}}{\left[\Phi^{-1}\left(1-\frac{\hat{s}_{\alpha}^{2 \text { hot }}}{2}\right)\right]^{-2}-\left[\Phi^{-1}\left(1-\frac{\hat{s}_{\alpha}^{2 \text { cold }}}{2}\right)\right]^{-2}}
\end{aligned}
$$


and an estimate of the correlation bias is a root of the following cubic:

$$
\begin{aligned}
\left(-\hat{r}_{a b}^{\text {hot }}+\frac{c_{0}^{\text {hot }}}{c_{0}^{\text {cold }}} \hat{r}_{a b}^{\text {cold }}\right) & \left(c_{1}^{\text {hot }}-\frac{c_{0}^{\text {hot }}}{c_{0}^{\text {cold }}}+c_{1}^{\text {cold }}\right) \hat{\rho}_{0} \\
& +\left(c_{3}^{\text {hot }}-\frac{c_{0}^{\text {hot }}}{c_{0}^{\text {cold }}} c_{3}^{\text {cold }}\right) \hat{\rho}_{0}^{3}=0
\end{aligned}
$$

The solution of a cubic equation is given in ([21], (3.8.2)). For this particular cubic there is typically one real root and a pair of complex conjugate roots. The real root is the desired solution for $\hat{\rho}_{0}$ and is given by:

$$
\hat{\rho}_{0}=\left[r+\left(q^{3}+r^{2}\right)^{\frac{1}{2}}\right]^{\frac{1}{3}}\left[r-\left(q^{3}+r^{2}\right)^{\frac{1}{2}}\right]^{\frac{1}{3}}
$$

where $q$ and $r$ are defined as

$$
\begin{array}{r}
q=\frac{1}{3} \frac{\left(c_{1}^{\text {hot }}-\frac{c_{0}^{\text {hot }}}{c_{0}^{\text {cold }}} c_{1}^{\text {cold }}\right)}{\left(c_{3}^{\text {hot }}-\frac{c_{0}^{\text {lot }}}{c_{0}^{\text {colc }}} c_{3}^{\text {cold }}\right)} \\
r=\frac{1}{2} \frac{\left(\hat{r}_{a b}^{\text {hot }}-\frac{c_{0}^{\text {hot }}}{c_{0}^{\text {cold }}} r_{a b}^{\text {cold }}\right)}{\left(c_{3}^{\text {hot }}-\frac{c_{0}^{\text {hot }}}{c_{0}^{\text {cold }}} c_{3}^{\text {cold }}\right)}
\end{array}
$$

Care must be taken in choosing the proper branch of the cube roots; otherwise the solution is straightforward.

Once $\hat{\pi}_{\delta}$ and $\hat{\rho}_{0}$ have been determined, the correlation coefficient estimate $\hat{\rho}$ is computed as follows:

$$
\begin{aligned}
\hat{\rho}=\left[\frac{1}{c_{1}}\left(\hat{r}_{a b}-\left.\hat{r}_{a b}\right|_{\rho=0}\right)-\frac{c_{3}}{c_{1}^{4}}\left(\hat{r}_{a b}-\left.\hat{r}_{a b}\right|_{\rho=0}\right)^{3}\right. \\
\left.+\left(3 \frac{c_{3}^{2}}{c_{1}^{7}}-\frac{c_{5}}{c_{1}^{6}}\right)\left(\hat{r}_{a b}-\left.\hat{r}_{a b}\right|_{\rho=0}\right)^{5}\right]-\hat{\rho}_{0}
\end{aligned}
$$

where the estimated digital correlation bias is computed as in (III-A1):

$$
\left.\hat{r}_{a b}\right|_{\rho=0}=\hat{\pi}_{\delta}\left(\frac{2}{\pi}\right) \theta_{a} \theta_{b} \exp \left[-\frac{1}{2}\left(\theta_{a}^{2}+\theta_{b}^{2}\right)\right]
$$

An estimate of the third Stokes parameter is subsequently computed using (5):

$$
\hat{T}_{U}=2 \hat{\rho} \sqrt{\hat{T}_{v, \mathrm{sys}} \hat{T}_{h, \mathrm{sys}}}
$$

\section{HARDWARE DEMONSTRATION}

The digital correlating polarimeter concept was demonstrated using the Polarimetric Scanning Radiometer/Digital (PSR/D) airborne imaging instrument [23]-[25]. The PSR/D is the first multi-channel tri-polarimetric (first three Stokes parameters) high-resolution imaging radiometer for aircraft-based studies of land and ocean emission (Fig. 9). The radiometer operated successfully in a conical scanning configuration to measure the first three Stokes parameters over the wind-driven ocean at $10.7 \mathrm{GHz}$ (X-band) and $37.0 \mathrm{GHz}$ (Ka-band) [1]. In-situ calibration was accomplished using unpolarized hot and ambient temperature blackbody calibration targets and verified using a ground-based polarimetric calibration target similar to that described in [8].

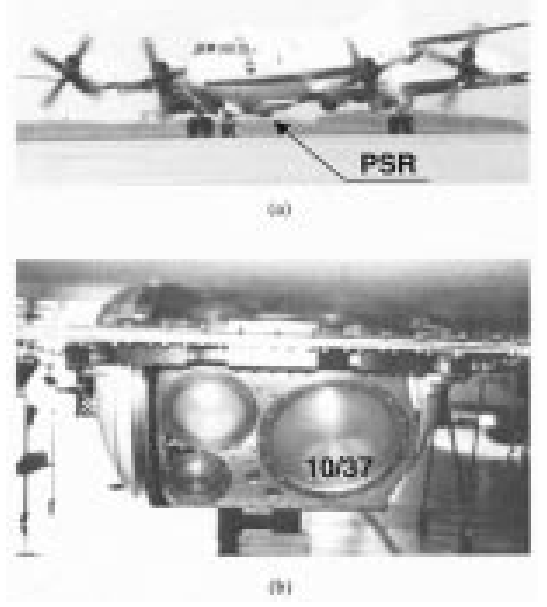

Fig. 9. (a) Polarimetric scanning radiometer (PSR) installed in the bomb bay of the NASA Wallops P-3B Orion (N426A). (b) PSR scanhead with antenna lenses visible. The dual-band $10.7 / 37.0 \mathrm{GHz}$ antenna is indicated.

\section{A. Hardware}

The $10.7 \mathrm{GHz}$ radiometer was a superheterodyne single-sideband (SSB) system with a low noise amplifier (LNA) front-end on each channel. The $37.0 \mathrm{GHz}$ radiometer was a superheterodyne double-sideband (DSB) system with mixer front-ends. The receiver topologies were similar to Fig. 1, with common local oscillators providing coherent downconversion of the two orthogonally-polarized channels. Sufficient RF and IF gain $(\sim 100 \mathrm{~dB})$ was provided to amplify the baseband signals to a level appropriate for three-level A/D conversion. A separate analog total-power detection circuit with square law detectors and video amplifiers was included. This redundant circuitry provided independent measurements of the first two Stokes parameters.

The IF signals of the $10.7 \mathrm{GHz}$ system were fed to a 1 GS/s emitter-coupled logic (ECL) digital correlator capable of measuring the variances and correlation coefficient of two 500 $\mathrm{MHz}$ orthogonally-polarized baseband random signals (see Fig. 10). The $37.0 \mathrm{GHz}$ system provided a $1000 \mathrm{MHz}$ wide IF band, thus, each IF band was subdivided into two $500 \mathrm{MHz}$ subbands. These signals were fed to two correlators. By such frequency demultiplexing, the entire available bandwidth of the $37.0 \mathrm{GHz}$ radiometer was utilized. Each correlator comprised three functional blocks: the A/D converters, the multipliers, and the accumulators. The high-speed A/D converters were constructed using dual-window comparators yielding three levels of quantization at a $1 \mathrm{GS} / \mathrm{s}$ rate. The comparator threshold levels were dynamically adjusted to track slowly varying drifts in IF signal power. Threshold level adjustment using eight-bit D/A converters provided (for Gaussian signals) a 48 $\mathrm{dB} A / \mathrm{D}$ converter input dynamic range. The operating dynamic range was limited to $\sim 30 \mathrm{~dB}$, however, by setting a minimum allowable threshold level to ensure a good SNR. The typical threshold level was $\sim 0.3 \mathrm{~V}$.

Estimates of second-order digital signal statistics were made by squaring and cross-multiplying the A/D converter outputs, 


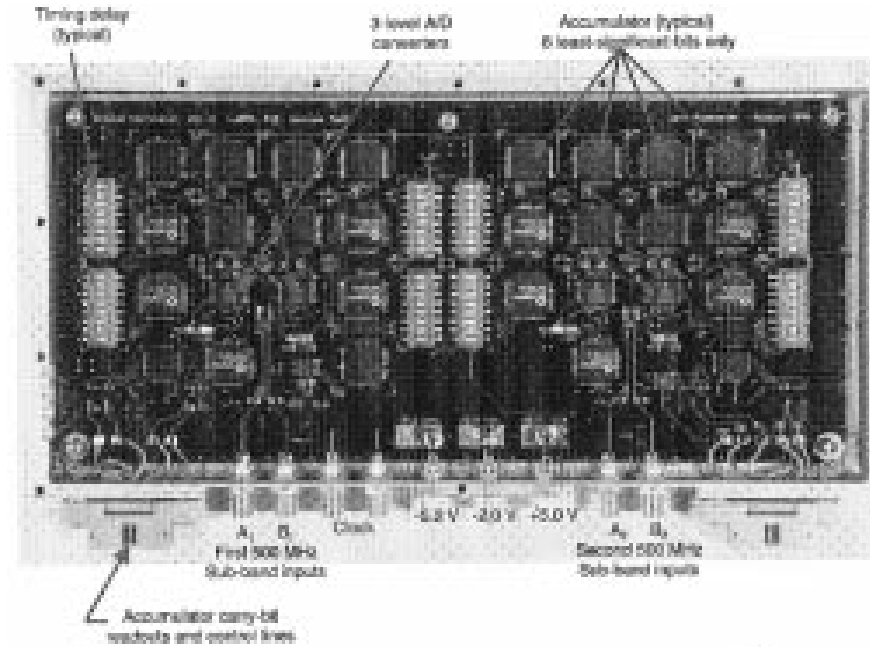

Fig. 10. Digital correlator module for polarimetric scanning radiometer (PSR/D). The correlator is constructed using ECL discrete logic on a six-layer microwave circuit board. This module contains two complete correlators (including total power channels) on one board.

then accumulating using digital counters. The total power, or variance, of an individual channel was measured by counting the number of times the input signal exceeded either the positive or negative threshold levels as in (9). The correlation coefficient was similarly determined by separately counting the number of positive and negative correlation counts. A total of eight AND/NAND gates composed the entire three-level multiplier circuit. The outputs of the digital multiplier were accumulated in four 24-bit counters providing $16.8 \mathrm{~ms}$ of integration time. The initial 1-Gbit multiplier outputs were prescaled using high-speed eight-bit ECL ripple counters. The system clock was distributed differentially to the counters using $50 \Omega$ odd-mode coupled microstrip lines. The high-speed ECL signals exhibit transition times shorter than $250 \mathrm{ps}$; therefore, the digital signals have spectral content $>4 \mathrm{GHz}$. On-chip and interconnect propagation delays within the multiplier circuit were compensated with clock delays generated by programmable delay chips. To save power, the output from these counters were carried to 16-bit TTL counters. The most-significant 16 bits were buffered and read by computer. The circuit was fabricated on six-layer G10 fiberglass circuit board. Microstrip interconnects were placed on the outer two layers of $1 / 2-\mathrm{oz}$ copper and power was distributed via the internal layers of 2-oz copper.

Redundant analog total-power channels were implemented in parallel with the digital radiometers. The same IF signals fed to the digital correlators were coupled to square-law detectors at $\sim-23 \mathrm{dBm}$ power level. Video amplifiers following the square-law detectors used integration times of $8 \mathrm{msec}$. The video amplifier output ranged from $0-10 \mathrm{~V}$ and was sampled by a 12-bit A/D converter. An analog offset was added to the video amplifier output to maintain the signal level within the operating voltage range of the $\mathrm{A} / \mathrm{D}$.

\section{B. Calibration}

The unpolarized hot and ambient method of Section IV was used to identify the correlation offset and the threshold-offset
TABLE I

Digital CORRElating POLARIMETER SySTEM PARAMETERS FOUND USING HOT AND AMBIENT UNPOLARIZED CALIBRATION TARGETS

\begin{tabular}{c|cc}
\hline Parameter & X-band $(10.6-10.8 \mathrm{GHz})$ & Ka-band $(36-38 \mathrm{GHz})$ \\
\hline$\sqrt{\hat{T}_{R E C, v, v} \hat{T}_{R E C, h}}(\mathrm{~K})$ & 1394 & 939 \\
$\hat{\rho}_{0}$ & 0.020 & 0.078 \\
$\hat{\pi}_{\delta}$ & 0.0024 & 0.0058 \\
Receiver type & LNA/SSB & mixer/DSB, two subbands \\
$\Delta T_{U, r m s}:$ Theory $(\mathrm{K})$ & 0.51 & 0.35 \\
$\Delta T_{U, r m s}:$ Meas'd (K) & 0.66 & 0.36 \\
\hline
\end{tabular}

product system parameters. Microwave foam absorber in ambient and liquid nitrogen conditions provided unpolarized radiation fields. The estimated system parameters and the radiometric sensitivities of the $T_{U}$ channels are presented in Table I. The radiometer sensitivities were measured by computing the standard deviation of 100 calibrated measurements while staring at an ambient temperature absorber. The resulting measured sensitivities are 1.03-1.29 times the fundamental limits stated in (26). The increased noise is attributed to RF and IF amplifier gain fluctuations, nonoptimal selection of threshold levels, hystersis, and threshold-level random noise.

To verify the effectiveness of the unpolarized two-look technique for calibrating the $T_{U}$ channel, a polarimetric calibration similar to that described in [8] was performed. The digitally-estimated third Stokes parameter $\hat{T}_{U}$, as given in Section IV, can be related to an incident polarized field by the following:

$$
\begin{aligned}
\hat{T}_{U} & =2\langle\hat{\rho}\rangle \sqrt{T_{\mathrm{sys}, v} T_{\mathrm{sys}, h}} \\
& =g_{U v} T_{v}+g_{U h} T_{h}+g_{U U} T_{U}+o_{U}
\end{aligned}
$$

where the gains $g_{U U}, g_{U v}$, and $g_{U h}$ and offset $o_{U}$ are unidentified system parameters that might have been left uncorrected by the two-look nonpolarized calibration procedure. Assuming that $\rho_{0}$ and $\pi_{\delta}$ completely calibrate the third Stokes parameter channel, then $g_{U U}=1$ and $g_{U v}=g_{U h}=o_{U}=0$ (the gain terms are dimensionless and $o_{U}$ is in K). By comparing measurements of $T_{U}$ with an expected polarized emission vector $\left[T_{v}, T_{h}, T_{U}\right]^{T}$ generated by a polarized calibration target, the residual gain and offset terms can be checked.

The polarized calibration target included an ambient load, a liquid nitrogen load, and a polarizing grid. The cold load was constructed by immersing a $56 \mathrm{~cm} \times 56 \mathrm{~cm}$ square of the convoluted foam absorber in liquid nitrogen. The liquid nitrogen bath covered the absorber tips by at least $0.5 \mathrm{~cm}$ to ensure temperature uniformity. The ambient load was shrouded in a styrofoam jacket. The temperature difference between the targets was $T_{\text {hot }}-T_{\text {cold }} \approx 210 \mathrm{~K}$. The polarized target was mounted on a turntable that provided rotation about the feedhorn axis. The rotational position was measured using an optical encoder with $0.25^{\circ}$ precision.

The details of the polarimetric calibration procedure are documented in [8]. Initially, the radiometer antenna was aligned with the polarized target such that the incident Stokes field was $T_{v}=T_{\text {cold }}, T_{h}=T_{\text {hot }}$ and $T_{U}=0$. Measurements were made while slowly rotating the target over an angular range of $\sim 420^{\circ}$. An additional piece of absorber at ambient temperature 


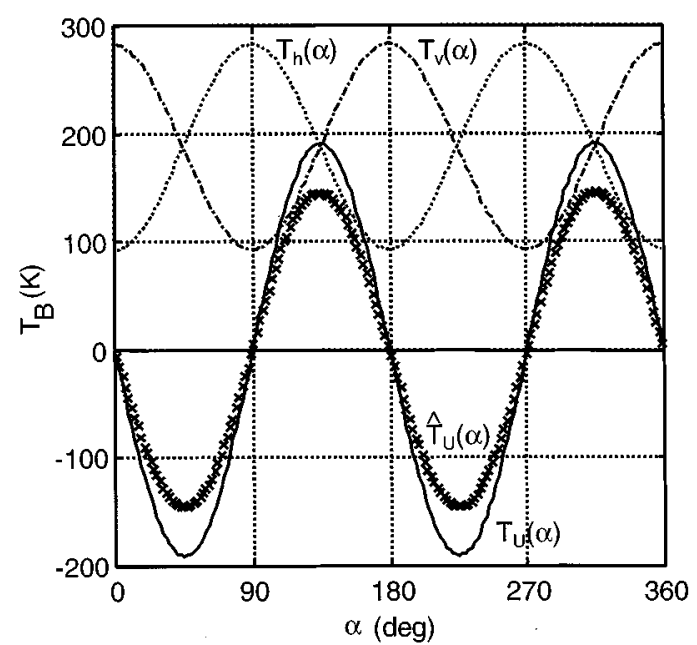

(a)

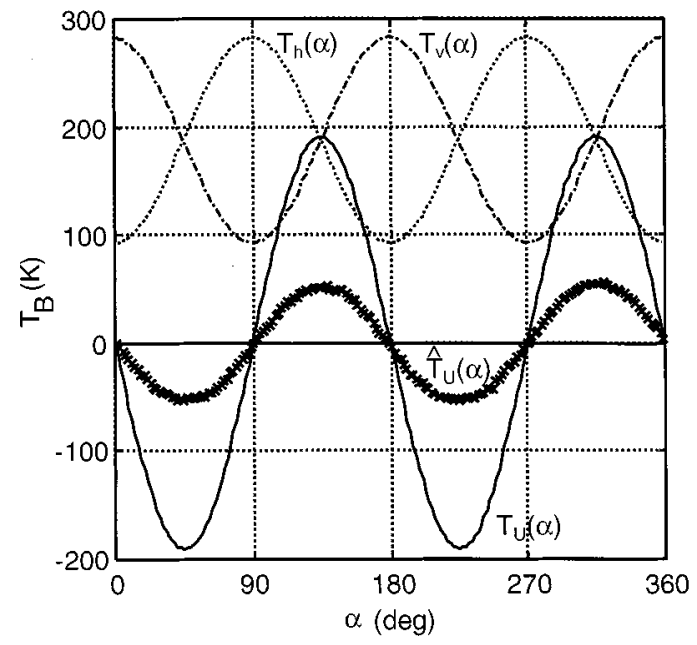

(b)

Fig. 11. Plots of (a) $37.0 \mathrm{GHz}$ and (b) $10.7 \mathrm{GHz}$ polarized target measurements $\hat{T}_{U}(\alpha)$ (denoted by crosses) and the observation matrix. The columns of the observation matrix are plotted as follows: $T_{v}(\alpha)$ (dash-dot), $T_{h}(\alpha)$ (dotted), and $T_{U}(\alpha)$ (solid).

was used for an unpolarized look. A total of 600 radiometer samples were recorded and averaged into $2^{\circ}$ bins, resulting in 180 points for a full $360^{\circ}$ rotation with $\sim 28 \mathrm{~ms}$ integration time per point $\left(\Delta T_{\mathrm{RMS}} \approx 0.4 \mathrm{~K}\right)$. Using the calibration parameters found with the two-look unpolarized method, the output of the digital correlator was converted into calibrated values of $\hat{T}_{U}\left(\alpha_{i}\right)(i=1 \ldots n)$ for the different angles $\alpha_{i}$ and $\hat{T}_{U}(U P)$ for the unpolarized look. The measurement vectors and the columns of the observation matrices for both 10.7 and $37.0 \mathrm{GHz}$ are plotted in Fig. 11. By visual inspection, $T_{v}$ and $T_{h}$ mixing into $\hat{T}_{U}$ appears to be nonexistent, but the correlator output is attenuated $\sim 25 \%$ at $37 \mathrm{GHz}$ and $\sim 70 \%$ at $10.7 \mathrm{GHz}$ compared to $T_{U}$.

Using these data, the gains and offsets for the 10.7 and 37.0 $\mathrm{GHz} T_{U}$ channels were calculated according to the methods in [8] and presented in Table II. First notable are the small $(\sim 0.1-0.05 \%) g_{U v}$ and $g_{U h}$ polarization-mixing gain terms. These negligible values are a direct result of using the digital correlator with the unpolarized calibration described in Section IV versus a polarization adding design. Second, the offsets $o_{U}$ are small $(-0.05 \mathrm{~K}$ and $0.44 \mathrm{~K})$, corresponding to correlation
TABLE II

GAIN AND OFFSET TERMS FOR THE 10.7 AND $37.0 \mathrm{GHz}$ Digital Polarimeters as MEASURED Using THE POLARIZED CALIBRATION STANDARD

\begin{tabular}{c|cc}
\hline & X-band $(10.7 \mathrm{GHz})$ & Ka-band $(37.0 \mathrm{GHz})$ \\
\hline$\widehat{g}_{U v}$ & $-2.32 \times 10^{-4}$ & $1.01 \times 10^{-3}$ \\
$\widehat{g}_{U h}$ & $3.90 \times 10^{-4}$ & $4.94 \times 10^{-4}$ \\
$\hat{g}_{U U}$ & 0.288 & 0.764 \\
$\hat{o}_{U}$ & -0.047 & -0.444 \\
\hline
\end{tabular}

TABLE III

COMPARISON OF Digital AND ANALOG TOTAL-POWER RADIOMETER MEASUREMENTS

\begin{tabular}{c|cc|cc}
\hline & \multicolumn{2}{|c|}{ X-band $(10.6-10.8 \mathrm{GHz})$} & \multicolumn{2}{|c}{ Ka-band $(36-38 \mathrm{GHz})$} \\
& v-pol & h-pol & v-pol & h-pol \\
\hline$\mu_{A-D}$ & -0.16 & -0.56 & -0.62 & -0.50 \\
$\sigma_{A-D}$ & 0.85 & 1.2 & 0.68 & 0.82 \\
$\sigma_{A}$ & 0.46 & 0.64 & 0.18 & 0.20 \\
$\sigma_{D}$ & 0.70 & 1.0 & 0.57 & 0.72 \\
$\sqrt{\sigma_{A}^{2}+\sigma_{D}^{2}}$ & 0.84 & 1.2 & 0.60 & 0.75 \\
\hline
\end{tabular}

coefficient offsets $\rho_{0} \sim 10^{-4}-10^{-5}$. Recall, this level of offset was the design goal setforth for systematic errors described Section III. The reasonable values of $g_{U v}, g_{U h}$, and $o_{U}$ demonstrate the successful application of the unpolarized two-look technique for calibrating the digital correlator.

We note, however, that the correlator gains $g_{U U}$ are significantly less than unity. This residual gain term cannot be identified using the unpolarized two-look technique because it does not provide a stimulus with known and nonzero correlation. The polarimetric calibration technique does provide this estimate. Sampler hysteresis accounts for a $\sim 5 \%$ attenuation. Timing skew and dispersion between the comparator-based A/D converters could account for up to $\sim 20 \%$ attenuation, with the remainder ascribed to phase-imbalances between mixers and image-rejection and low-pass filters present in the radiometers. Subsequent polarimetric calibration experiments have shown that this attenuation does not vary by more than a few percent over several months, allowing a constant correlator gain coefficient to be included in the postflight data processing routines.

The calibration exercise also yielded analog and digital total-power measurements that were compared for consistency. One hundred twenty-five samples with $T_{B}=80$ to $290 \mathrm{~K}$ were compared. The mean and standard deviation of the analog-digital measurement differences are tabulated in Table III. The mean of the differences $\mu_{\mathrm{A}-\mathrm{D}}$ are relatively small and range from -0.16 to $-0.62 \mathrm{~K}$. The constant negative bias across all four channels suggests a slight nonlinearity of $\sim 0.5 \mathrm{~K}$ over a $\sim 200 \mathrm{~K}$ range or $\sim 0.25 \%$ full scale. The standard deviations of the analog and digital data are denoted $\sigma_{A}$ and $\sigma_{D}$, respectively, and are equivalent to their respective $\Delta T_{\mathrm{RMSs}}$. The standard deviation of the set of differenced brightnesses $\sigma_{\mathrm{A}-\mathrm{D}}$ is close to the root-sum-squares $\left(\sqrt{\sigma_{A}^{2}+\sigma_{D}^{2}}\right)$ of the individual standard deviations. This consistency is important because it indicates that the variations in the differenced data are produced by the independant random variations in the individual analog and digital data sets as opposed to some correlated, external, random fluxations affecting both analog and digital detectors. 

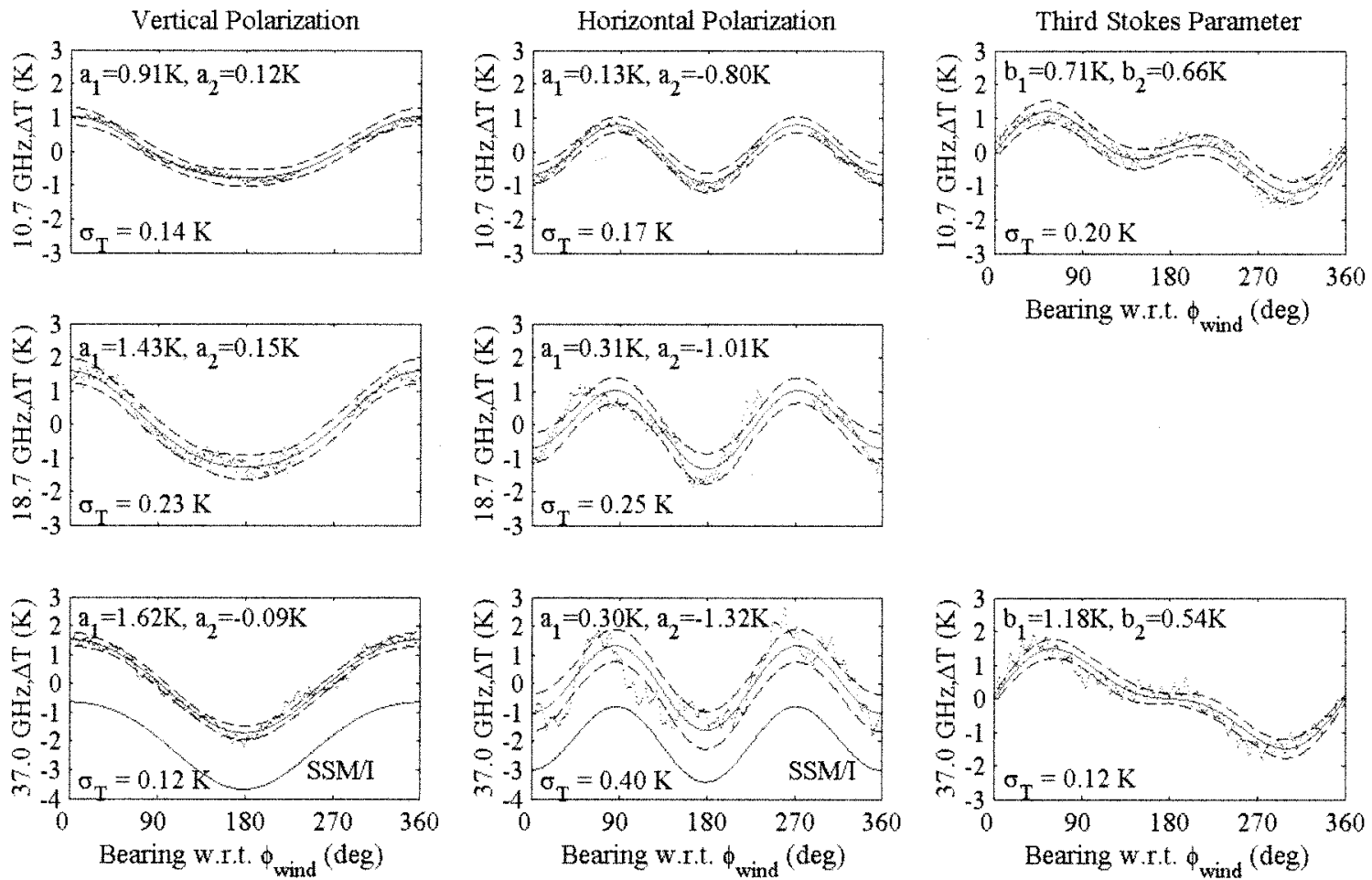

Fig. 12. PSR azimuthal harmonics exhibiting wind direction dependence of the first three Stokes parameters at 10.7, 18.7, and 37.0 GHz. Data for $T_{U}$ at 18.7 GHz was unavailable. The wind speed was $\sim 14 \mathrm{~ms}^{-1}$ from 1500 to 1600 UTC on March 4, 1997. The solid lines represent the reconstructed second-order harmonic expansions and the dashed lines are the $\pm 1 \sigma_{T}$ error curves for 135 scans. Individual points indicate mean measured brightness deviations. The $37.0 \mathrm{GHz}$ SSM/I global average wind direction harmonics, denoted by solid lines, are shifted by $-2 \mathrm{~K}$ for clarity.

In all, the digital total-power radiometer tracks the analog system quite well.

\section{Ocean Surface Emission Measurements}

Perhaps the most important application of the third Stokes parameter is the measurement of ocean surface wind vectors (e.g., [1]). Boundary layer winds drive the ocean surface creating gravity and capillary waves and ocean foam, which in turn cause azimuthal dependencies in the upwelling microwave brightness temperatures [26], [27]. This dependence has been observed using both satellite radiometers [28] in $T_{v}$ and $T_{h}$ aircraft radiometers [29]-[31] in all four components of the Stokes vector.

In March 1997, the PSR/D, along with a complement of passive and active instruments (together known as the Ocean Winds Imaging suite, or OWI [30]), was operated aboard the NASA Wallops Flight Facility P-3B aircraft as part of the Labrador Sea Deep Convection Experiment. Conically scanned microwave brightness imagery of the wind driven ocean surface was collected during coordinated flights over the R.V. Knorr. We discuss here PSR/D data collected on March 4, 1997 from 15:00-16:00 UTC. Centered over the Knorr, the flight pattern consisted of six straight and level flight legs organized in three pairs, each $60^{\circ}$ apart in heading. This "hex-cross" pattern covered a triangular-shaped area $\sim 30 \mathrm{~km}$ along a side. Data collected during the pattern consisted of 135 scans with 227 samples per scan at an incidence angle of $53.1^{\circ}$ from nadir. Surface winds were $\sim 14 \mathrm{~ms}^{-1}$ from $\sim 260^{\circ}$ as measured by both GPS dropsonde and Knorr wind sensors. The ocean temperature measured at $h=-3 \mathrm{~m}$ was $3^{\circ} \mathrm{C}$, and the air temperature at $h=23 \mathrm{~m}$ was $-12{ }^{\circ} \mathrm{C}$.

The measured azimuthal brightness variations at 10.7, 18.7, and $37.0 \mathrm{GHz}$ are plotted in Fig. 12. The 135 scans were averaged to accumulate a total of $\sim 2.2 \mathrm{~s}$ of integration time for each of the 227 azimuthal points, thus corresponding to a $\Delta T_{\text {rms }}$ of $\sim 0.05 \mathrm{~K}$. Averaging over $\sim 30 \mathrm{~km}$ of ocean surface simulates satellite footprint conditions. First and second-order harmonic amplitudes were determined via a least-squares fit to the mean azimuthal signature and are overlaid in the plot. For comparison, the $37 \mathrm{GHz}$ Special Sensor Microwave/Imager (SSM/I) global average wind direction harmonics from [28] are also plotted with a $-2 \mathrm{~K}$ offset for clarity. The plots show a distinct variation of $\sim 2-4 \mathrm{~K}$ with strong first and second harmonic dependence in the vertical and horizontal polarizations, respectively. For both polarizations, the dominant harmonic amplitude is larger at the higher frequency. Furthermore, the measured vertical and horizontal harmonic amplitudes at $37.0 \mathrm{GHz}$ exhibit excellent agreement with the SSM/I global average wind direction harmonics. Although slightly lower in amplitude, the PSR 10.7 $\mathrm{GHz}$ wind direction harmonics are otherwise comparable to the $37 \mathrm{GHz}$ SSM/I harmonics. Of particular interest is the strong ( $\sim 1 \mathrm{~K}$ amplitude) first harmonic present in the third Stokes parameter signature. The large first harmonic content of this signature is indicative of a strong windward-leeward asymmetry in the ocean wave structure. The measurements are similar to simulated results obtained using an asymmetric wave geometrical optics model [26]. These measurements and their excellent 
agreement with satellite and model data verify the utility, calibration technique, and performance capability of the digital polarimetric radiometer.

\section{DISCUSSION}

The design techniques and radiometer hardware described here demonstrate the utility and technological feasibility of the digital polarimetric radiometer for earth remote sensing applications. Other polarimeter topologies, such as adding polarimeters, are possibilities. Such systems, however, can exhibit Stokes parameter mixing beyond that caused by the antenna system that is not easily identifiable without sophisticated calibration techniques. On the contrary, the digital polarimeter has the distinct advantage of negligible Stokes parameter mixing using unpolarized calibration. Further, use of a three-level digital correlator provides a simple means of calibrating both correlation offsets as well as total power measurements.

To reiterate, the following design rules developed in Section III for the A/D converter parameters required to limit offset and gain perturbations are

1) $v_{\delta_{\alpha}}<0.01 \sigma_{v_{\alpha}}$ to minimize correlator offsets $\left.r_{a b}\right|_{\rho=0}$ and $\delta r_{a b}$

2) minimize hysteresis, timing skew, and phase differences to maximize $g_{U U}$.

Adherence to these rules is highly desirable in order that the radiometer can be used to make accurate measurements of the third and fourth Stokes parameters. However, if these design specifications cannot be met, the radiometer may still be used pending regular calibration using a polarimetric calibration standard or a similar method such as correlated noise injection to compensate for gain and offset variations.

The PSR/D hardware demonstration confirms the ability to fabricate, operate, and calibrate a digital polarimetric radiometer in the field and exemplifies its utility in earth remote sensing, particularly in the observation of ocean surface winds. As a follow-on to the PSR/D demonstration, an implementation suitable for satellite deployment using lower-power space-qualified CMOS logic at comparable sample rates is feasible and is currently under development. An incidental consequence of this work also exists in the application of digital correlators for synthetic aperture interferometric radiometry (e.g., [32]). Such systems will be susceptible to the same effects of nonlinearity, threshold asymmetry, timing skew, A/D converter hysteresis, and phase errors, all of which have been discussed here.

\section{APPENDIX I}

\section{CORRELATION COEFFICIENT INVERSION}

The digital correlation coefficient can be computed by the following:

$$
\begin{aligned}
r_{a b}= & \sigma_{v_{a}} \sigma_{v_{b}} \int_{0}^{\rho} f\left(\sigma_{v_{a}} \theta_{a}, \sigma_{v_{b}} \theta_{b} ; \rho^{\prime}\right) \\
& +f\left(\sigma_{v_{a}} \theta_{a},-\sigma_{v_{b}} \theta_{b} ; \rho^{\prime}\right)+f\left(-\sigma_{v_{a}} \theta_{a}, \sigma_{v_{b}} \theta_{b} ; \rho^{\prime}\right) \\
& +f\left(-\sigma_{v_{a}} \theta_{a},-\sigma_{v_{b}} \theta_{b} ; \rho^{\prime}\right) d \rho^{\prime} .
\end{aligned}
$$

Symmetry of the Gaussian pdf allows us to write

$$
\begin{aligned}
r_{a b}=2 \sigma_{v_{a}} \sigma_{v_{b}} \int_{0}^{\rho} f\left(\sigma_{v_{a}} \theta_{a}, \sigma_{v_{b}} \theta_{b} ; \rho^{\prime}\right) \\
\\
+f\left(\sigma_{v_{a}} \theta_{a},-\sigma_{v_{b}} \theta_{b} ; \rho^{\prime}\right) d \rho^{\prime} .
\end{aligned}
$$

The function $\sigma_{v_{a}} \sigma_{v_{b}} f\left(\sigma_{v_{a}} \theta_{a}, \sigma_{v_{b}} \theta_{b} ; \rho\right)$ is recognized as the bivariate normal pdf $[21,(26.3 .1)]$

$$
p\left(\theta_{a}, \theta_{b} ; \rho\right)=\frac{1}{2 \pi \sqrt{1-\rho^{2}}} \exp \left[-\frac{\theta_{a}^{2}-2 \rho \theta_{a} \theta_{b}+\theta_{b}^{2}}{2\left(1-\rho^{2}\right)}\right] .
$$

Rewriting the expression for $r_{a b}$ using the bivariate normal pdf yields

$$
r_{a b}=2 \int_{0}^{\rho} p\left(\theta_{a}, \theta_{b} ; \rho^{\prime}\right)+p\left(\theta_{a},-\theta_{b} ; \rho^{\prime}\right) d \rho^{\prime}
$$

The task at hand is to expand the integrand in a Taylor series and then integrate. The integrand of the previous is

$$
I\left(\rho^{\prime}\right)=2 p\left(\theta_{a}, \theta_{b} ; \rho^{\prime}\right)+2 p\left(\theta_{a},-\theta_{b} ; \rho^{\prime}\right) .
$$

This can be expanded in a Taylor series in terms of $\rho^{\prime}$

$$
\begin{aligned}
I\left(\rho^{\prime}\right)=I(0)+ & I^{(1)}(0) \rho^{\prime}+\frac{1}{2 !} I^{(2)}(0) \rho^{2} \\
& +\frac{1}{3 !} I^{(3)}(0) \rho^{3}+\frac{1}{4 !} I^{(4)}(0) \rho^{4}+\cdots
\end{aligned}
$$

The algebra involving the derivatives is quite cumbersome and the computer algebra package MapleV was used to evaluate the derivatives. These derivatives are

$$
\begin{aligned}
\left.\frac{\partial}{\partial \rho} p\left(\theta_{a}, \theta_{b}, \rho\right)\right|_{\rho=0} & =p\left(\theta_{a}, \theta_{b}, 0\right) \theta_{a} \theta_{b} \\
\left.\frac{\partial^{2}}{\partial \rho^{2}} p\left(\theta_{a}, \theta_{b}, \rho\right)\right|_{\rho=0}= & p\left(\theta_{a}, \theta_{b}, 0\right)\left(\theta_{a}^{2}-1\right)\left(\theta_{b}^{2}-1\right) \\
\left.\frac{\partial^{3}}{\partial \rho^{3}} p\left(\theta_{a}, \theta_{b}, \rho\right)\right|_{\rho=0}= & p\left(\theta_{a}, \theta_{b}, 0\right)\left(3 \theta_{a}-\theta_{a}^{3}\right)\left(3 \theta_{b}-\theta_{b}^{3}\right) \\
\left.\frac{\partial^{4}}{\partial \rho^{4}} p\left(\theta_{a}, \theta_{b}, \rho\right)\right|_{\rho=0}= & p\left(\theta_{a}, \theta_{b}, 0\right)\left(3-6 \theta_{a}^{2}+\theta_{a}^{4}\right) \\
& \times\left(3-6 \theta_{b}^{2}+\theta_{b}^{4}\right) .
\end{aligned}
$$

The derivatives of $p\left(\theta_{a},-\theta_{b}, \rho\right)$ are easily found by substituting $-\theta_{b}$ for $\theta_{b}$ in the previous equation. Because the first and third derivatives are odd functions of $\theta_{a}$ and $\theta_{b}$, it is immediately seen that the Taylor series terms with odd powers of $\rho$ will cancel leaving only the even powers of $\rho$. Adding the appropriate derivatives yields the following for the integrand:

$$
\begin{aligned}
& I\left(\rho^{\prime}\right)=4 p\left(\theta_{a}, \theta_{b}, 0\right)\left[1+\frac{1}{2}\left(\theta_{a}^{2}-1\right)\left(\theta_{b}^{2}-1\right) \rho^{\prime 2}\right. \\
& \left.+\frac{1}{24}\left(3-6 \theta_{a}^{2}+\theta_{a}^{4}\right)\left(3-6 \theta_{b}^{2}+\theta_{b}^{4}\right) \rho^{4}\right]+\mathcal{O}\left(\rho^{\prime 6}\right) .
\end{aligned}
$$


Finally, integrating the previous yields

$$
\begin{aligned}
r_{a b}= & \frac{2}{\pi} \exp \left[-\frac{1}{2}\left(\theta_{a}^{2}+\theta_{b}^{2}\right)\right] \\
& \times\left[\rho+\frac{1}{6}\left(\theta_{a}^{2}-1\right)\left(\theta_{b}^{2}-1\right) \rho^{3}+\frac{1}{120}\left(3-6 \theta_{a}^{2}+\theta_{a}^{4}\right)\right. \\
& \left.\times\left(3-6 \theta_{b}^{2}+\theta_{b}^{4}\right) \rho^{5}\right]+\mathcal{O}\left(\rho^{7}\right) .
\end{aligned}
$$

\section{APPENDIX II \\ DIGITAL RADIOMETER SENSITIVITY}

This appendix contains a derivation of the sensitivities of both the cross- and autocorrelating channels of the digital polarimeter. These sensitivities are assumed to be optimized with respect to the $\mathrm{A} / \mathrm{D}$ converter threshold level.

\section{A. Cross-Correlator Sensitivity}

The sensitivity of the third Stokes parameter cross-correlating channel is

$$
\Delta T_{U, \mathrm{rms}}=\frac{\sigma_{\hat{r}_{a b}}}{\partial r_{a b} / \partial T_{U}}
$$

Using the chain rule, the derivative in the denominator is expanded

$$
\frac{\partial r_{a b}}{\partial T_{U}}=\frac{\partial r_{a b}}{\partial \rho} \frac{\partial \rho}{\partial T_{U}}
$$

The derivative $\partial r_{a b} / \partial \rho$ evaluated for small $\rho$ can be computed using (15) and (16)

$$
\begin{aligned}
\lim _{\rho \rightarrow 0} \frac{\partial r}{\partial \rho}= & \lim _{\rho \rightarrow 0} \sigma_{v_{a}} \sigma_{v_{b}}\left[f\left(\sigma_{v_{a}} \theta_{a}, \sigma_{v_{b}} \theta_{b} ; \rho\right)\right. \\
& +f\left(-\sigma_{v_{a}} \theta_{a}, \sigma_{v_{b}} \theta_{b} ; \rho\right)+f\left(\sigma_{v_{a}} \theta_{a},-\sigma_{v_{b}} \theta_{b} ; \rho\right) \\
& \left.+f\left(-\sigma_{v_{a}} \theta_{a},-\sigma_{v_{b}} \theta_{b} ; \rho\right)\right] \\
= & \lim _{\rho \rightarrow 0} 2 \sigma_{v_{a}} \sigma_{v_{b}}\left[f\left(\sigma_{v_{a}} \theta_{a}, \sigma_{v_{b}} \theta_{b} ; \rho\right)\right. \\
& \left.+f\left(-\sigma_{v_{a}} \theta_{a}, \sigma_{v_{b}} \theta_{b} ; \rho\right)\right] .
\end{aligned}
$$

For the typical correlator, the channels are assumed to be balanced (i.e., $\sigma_{v_{a}}=\sigma_{v_{b}}=\sigma$ ), and the threshold levels for both of the input $\mathrm{A} / \mathrm{D}$ converters are assumed to be equal (i.e., $\theta_{a}=\theta_{b}=\theta$ ). Thus, the previous equation is simplified

$$
\begin{aligned}
\lim _{\rho \rightarrow 0} \frac{\partial r}{\partial \rho} & =\lim _{\rho \rightarrow 0} 2 \sigma^{2}[f(\sigma \theta, \sigma \theta ; \rho)+f(-\sigma \theta, \sigma \theta ; \rho)] \\
& =2 \sigma^{2}[f(\sigma \theta, \sigma \theta ; 0)+f(-\sigma \theta, \sigma \theta ; 0)] \\
& =4 \sigma^{2} f(\sigma \theta, \sigma \theta ; 0) .
\end{aligned}
$$

The remaining derivative is obtained from (5)

$$
\frac{\partial \rho}{\partial T_{U}}=\frac{1}{2 \sqrt{T_{v, \mathrm{sys}} T_{h, \mathrm{sys}}}}
$$

In the numerator of (83), the standard deviation of $\hat{r}_{a b}$ is found by definition

$$
\sigma_{\hat{r}_{a b}}=\sqrt{\left\langle\hat{r}_{a b}^{2}\right\rangle-\left\langle\hat{r}_{a b}\right\rangle^{2}}
$$

Under the limiting case of $\rho \rightarrow 0$, the expected value of the digital covariance is zero

$$
\lim _{\rho \rightarrow 0}\left\langle\hat{r}_{a b}\right\rangle=0 .
$$

Thus, the standard deviation now is

$$
\lim _{\rho \rightarrow 0} \sigma_{\hat{r}_{a b}}=\lim _{\rho \rightarrow 0} \sqrt{\left\langle\hat{r}_{a b}^{2}\right\rangle}
$$

Expanding the quantity $\left\langle\hat{r}_{a b}^{2}\right\rangle$ results in

$$
\begin{aligned}
\lim _{\rho \rightarrow 0}\left\langle\hat{r}_{a b}^{2}\right\rangle= & \lim _{\rho \rightarrow 0}\left\langle\left[\frac{1}{N} \sum_{n=1}^{N} h\left(v_{a}(n T)\right) h\left(v_{b}(n T)\right)\right]\right. \\
& \left.\times\left[\frac{1}{N} \sum_{m=1}^{N} h\left(v_{a}(m T)\right) h\left(v_{b}(m T)\right)\right]\right\rangle \\
= & \lim _{\rho \rightarrow 0}\left\langle\frac{1}{N^{2}} \sum_{n=1}^{N} \sum_{m=1}^{N} h\left(v_{a}(n T)\right) \cdot h\left(v_{b}(n T)\right)\right. \\
& \left.\cdot h\left(v_{a}(m T)\right) \cdot h\left(v_{b}(m T)\right)\right\rangle \\
= & \lim _{\rho \rightarrow 0} \frac{1}{N^{2}} \sum_{n=1}^{N} \sum_{m=1}^{N}\left\langle h\left(v_{a}(n T)\right) \cdot h\left(v_{b}(n T)\right)\right. \\
& \left.\cdot h\left(v_{a}(m T)\right) \cdot h\left(v_{b}(m T)\right)\right\rangle
\end{aligned}
$$

Evaluating the limit allows the expected value within the double sum to be separated into two parts because $v_{a}$ and $v_{b}$ are statistically independent when $\rho \rightarrow 0$

$$
\begin{aligned}
\lim _{\rho \rightarrow 0}\left\langle\hat{r}_{a b}^{2}\right\rangle=\frac{1}{N^{2}} \sum_{n=1}^{N} \sum_{m=1}^{N}\langle & \left.h\left(v_{a}(n T)\right) h\left(v_{a}(m T)\right)\right\rangle \\
& \times\left\langle h\left(v_{b}(n T)\right) h\left(v_{b}(m T)\right)\right\rangle .
\end{aligned}
$$

Furthermore, because the samples are independent and identically distributed, all nonzero-lag products are zero: $\left\langle h\left(v_{a}(n T)\right) h\left(v_{a}(m T)\right)\right\rangle=0$ for $n \neq m$. Thus, the previous double summation becomes

$$
\begin{aligned}
\lim _{\rho \rightarrow 0}\left\langle\hat{r}_{a b}^{2}\right\rangle & =\frac{1}{N^{2}} \sum_{n=1}^{N}\left\langle h^{2}\left(v_{a}(n T)\right)\right\rangle\left\langle h^{2}\left(v_{b}(n T)\right)\right\rangle \\
& =\frac{1}{N}\left\langle h^{2}\left(v_{a}\right)\right\rangle\left\langle h^{2}\left(v_{b}\right)\right\rangle \\
& =\frac{1}{N}\left\langle\hat{s}_{a}^{2}\right\rangle\left\langle\hat{s}_{b}^{2}\right\rangle .
\end{aligned}
$$

Finally, by combining the previous results, the radiometric sensitivity is found

$$
\lim _{\rho \rightarrow 0} \Delta T_{U, \mathrm{rms}}=\frac{\sqrt{\left\langle\hat{s}_{a}^{2}\right\rangle\left\langle\hat{s}_{b}^{2}\right\rangle T_{v, \mathrm{sys}} T_{h, \mathrm{sys}}}}{2 \sigma^{2} f(\sigma \theta, \sigma \theta ; 0) \sqrt{N}} .
$$

The previous expression can be written in terms of $\theta$ by substituting in (7) and (11)

$$
\lim _{\rho \rightarrow 0} \Delta T_{U, \mathrm{rms}}=\frac{2 \pi e^{\theta^{2}}}{\sqrt{N}}[1-\Phi(\theta)] \sqrt{T_{v, \mathrm{sys}} T_{h, \mathrm{sys}}} .
$$

Computing the value of $\theta$ for the minimum $\Delta T_{U \text {,rms }}$ can be done using Newton's method. The optimal $\theta$ is 0.61 with

$$
\Delta T_{U, \mathrm{rms}}=\frac{2.47}{\sqrt{N}} \sqrt{T_{v, \mathrm{sys}} T_{h, \mathrm{sys}}} .
$$


Comparing this to the continuous correlator

$$
\Delta T_{U, \mathrm{rms}}=\frac{2}{\sqrt{N}} \sqrt{T_{v, \mathrm{sys}} T_{h, \mathrm{sys}}} .
$$

\section{B. Total-Power Sensitivity}

The sensitivity of the total-power channel is found similarly

$$
\Delta T_{\alpha, \mathrm{rms}}=\frac{\sqrt{\left\langle\left(\hat{s}_{\alpha}^{2}\right)^{2}\right\rangle-\left\langle\hat{s}_{\alpha}^{2}\right\rangle^{2}}}{\partial\left\langle\hat{s}_{\alpha}^{2}\right\rangle / \partial T_{\mathrm{ANT}, \alpha}} .
$$

Once again, the denominator is expanded using the chain rule

$$
\frac{\partial\left\langle\hat{s}_{\alpha}^{2}\right\rangle}{\partial T_{\mathrm{ANT}, \alpha}}=\frac{\partial\left\langle\hat{s}_{\alpha}^{2}\right\rangle}{\partial\left(\sigma_{v_{\alpha}}^{2}\right)} \frac{\partial\left(\sigma_{v_{\alpha}}^{2}\right)}{\partial T_{\mathrm{ANT}, \alpha}} .
$$

The first term in the product is the differential relationship between the input voltage variance and the output of the totalpower channel of the digital correlator. From (11)

$$
\begin{aligned}
\frac{\partial\left\langle\hat{s}_{\alpha}^{2}\right\rangle}{\partial\left(\sigma_{v_{\alpha}}^{2}\right)} & =2 \frac{\partial}{\partial\left(\sigma_{v_{\alpha}}^{2}\right)}\left[1-\Phi\left(\frac{v_{\mathrm{th}, \alpha}}{\sqrt{\sigma_{v_{\alpha}}^{2}}}\right)\right] \\
& =\frac{v_{\mathrm{th}, \alpha}}{\sigma_{v_{\alpha}}^{3} \sqrt{2 \pi}} \exp \left[-\frac{1}{2}\left(\frac{v_{\mathrm{th}, \alpha}}{\sigma_{v_{\alpha}}}\right)^{2}\right] .
\end{aligned}
$$

Recalling (14), the variance of the input voltage signal is

$$
\sigma_{v_{\alpha}}^{2}=R_{0} k B G_{\alpha}\left(T_{\mathrm{ANT}, \alpha}+T_{\mathrm{REC}, \alpha}\right)
$$

so that

$$
\frac{\partial\left(\sigma_{v_{\alpha}}^{2}\right)}{\partial T_{\mathrm{ANT}, \alpha}}=\frac{\sigma_{v_{\alpha}}^{2}}{T_{\mathrm{sys}, \alpha}}
$$

where $T_{\mathrm{sys}, \alpha}=T_{\mathrm{REC}, \alpha}+T_{\mathrm{ANT}, \alpha}$. Combining the two derivatives yields

$$
\begin{aligned}
\frac{\partial\left\langle\hat{s}_{\alpha}^{2}\right\rangle}{\partial T_{\mathrm{ANT}, \alpha}} & =\frac{1}{T_{\mathrm{sys}, \alpha} \sqrt{2 \pi}}\left(\frac{v_{\mathrm{th}, \alpha}}{\sigma_{v_{\alpha}}}\right) \exp \left[-\frac{1}{2}\left(\frac{v_{\mathrm{th}, \alpha}}{\sigma_{v_{\alpha}}}\right)^{2}\right] \\
& =\frac{1}{T_{\mathrm{sys}, \alpha} \sqrt{2 \pi}} \theta_{\alpha} e^{-\frac{1}{2} \theta_{\alpha}^{2}} .
\end{aligned}
$$

In the numerator of (103), the standard deviation of $\hat{s}_{\alpha}^{2}$ is found by definition

$$
\sqrt{\left\langle\left(\hat{s}_{\alpha}^{2}\right)^{2}\right\rangle-\left\langle\hat{s}_{\alpha}^{2}\right\rangle^{2}}
$$

This is computed by first expanding the expected value of $\left(\hat{s}_{\alpha}^{2}\right)^{2}$

$$
\begin{aligned}
\left\langle\left(\hat{s}_{\alpha}^{2}\right)^{2}\right\rangle & =\left\langle\left[\frac{1}{N} \sum_{n=1}^{N} h^{2}\left(v_{\alpha}(n T)\right)\right]\right. \\
& \left.\times\left[\frac{1}{N} \sum_{m=1}^{N} h^{2}\left(v_{\alpha}(m T)\right)\right]\right\rangle \\
& =\left\langle\frac{1}{N^{2}} \sum_{n=1}^{N} \sum_{m=1}^{N} h^{2}\left(v_{\alpha}(n T)\right) h^{2}\left(v_{\alpha}(m T)\right)\right\rangle \\
& =\frac{1}{N^{2}} \sum_{n=1}^{N} \sum_{m=1}^{N}\left\langle h^{2}\left(v_{\alpha}(n T)\right) h^{2}\left(v_{\alpha}(m T)\right)\right\rangle
\end{aligned}
$$

Because the samples of $v_{\alpha}$ are independent and identically distributed, the above expected value can be written

$$
\begin{aligned}
& \left\langle h^{2}\left(v_{\alpha}(n T)\right) h^{2}\left(v_{\alpha}(m T)\right)\right\rangle \\
& \quad= \begin{cases}\left\langle h^{2}\left(v_{\alpha}(n T)\right)\right\rangle\left\langle h^{2}\left(v_{\alpha}(m T)\right)\right\rangle, & \text { for } n \neq m \\
\left\langle h^{4}\left(v_{\alpha}(n T)\right)\right\rangle, & \text { for } n=m\end{cases}
\end{aligned}
$$

Conveniently, by the definition of $h(v), h^{4}(v)=h^{2}(v)$. Substituting these two cases into the double summation yields

$$
\begin{aligned}
\left\langle\left(\hat{s}_{\alpha}^{2}\right)^{2}\right\rangle= & \frac{1}{N^{2}} \sum_{n=1}^{N}\left\langle h^{2}\left(v_{\alpha}(n T)\right)\right\rangle \\
& \left.+\frac{1}{N^{2}} \sum_{\substack{n=1 \\
n \neq m=1}}^{N} \sum_{\substack{m=m \\
N}}^{N}\left(h^{2}(n T)\right)\right\rangle\left\langle h^{2}\left(v_{\alpha}(m T)\right)\right\rangle \\
= & \frac{1}{N}\left\langle\hat{s}_{\alpha}^{2}\right\rangle+\left(1-\frac{1}{N}\right)\left\langle\hat{s}_{\alpha}^{2}\right\rangle^{2}
\end{aligned}
$$

Thus, the variance of $\hat{s}_{\alpha}^{2}$ is

$$
\begin{aligned}
& \sqrt{\left\langle\left(\hat{s}_{\alpha}^{2}\right)^{2}\right\rangle-\left\langle\hat{s}_{\alpha}^{2}\right\rangle^{2}} \\
& =\sqrt{\frac{1}{N}\left\langle\hat{s}_{\alpha}^{2}\right\rangle+\left(1-\frac{1}{N}\right)\left\langle\hat{s}_{\alpha}^{2}\right\rangle^{2}-\left\langle\hat{s}_{\alpha}^{2}\right\rangle^{2}} \\
& =\sqrt{\frac{1}{N}\left\langle\hat{s}_{\alpha}^{2}\right\rangle\left(1-\left\langle\hat{s}_{\alpha}^{2}\right\rangle\right)} \\
& =\sqrt{\frac{1}{N}\left[1-\Phi\left(\theta_{\alpha}\right)\right] \Phi\left(\theta_{\alpha}\right) .}
\end{aligned}
$$

Forming the quotient (98) with the variance (108) and the derivative (103) produces

$$
\Delta T_{\alpha, \mathrm{rms}}=\frac{\sqrt{2 \pi}}{\theta_{\alpha}} e^{\frac{1}{2} \theta_{\alpha}^{2}} \sqrt{\Phi\left(\theta_{\alpha}\right)-\Phi^{2}\left(\theta_{\alpha}\right)} \frac{T_{\mathrm{sys}, \alpha}}{\sqrt{N}}
$$

The optimum value of $\theta_{\alpha}$ for best total power radiometer sensitivity is found by minimizing $\Delta T_{\alpha, \mathrm{rms}}$ with respect to $\theta_{\alpha}$. Again, this can be done numerically. The sensitivity is minimized at $\theta_{\alpha}=1.58$ such that

$$
\Delta T_{\alpha, \mathrm{rms}}=1.28 \frac{T_{\mathrm{sys}, \alpha}}{\sqrt{N}} .
$$

For operation of the polarization correlating radiometer, however, the value of $\theta_{\alpha}$ is set to minimize the noise of the $T_{U}$ measurement. At the optimal point for $T_{U}$ where $\theta_{\alpha}=0.61$, the sensitivity of the total power channel is

$$
\Delta T_{\alpha, \mathrm{rms}}=2.20 \frac{T_{\mathrm{sys}, \alpha}}{\sqrt{N}}
$$

\section{APPENDIX III}

\section{THRESHOLD OFFSET EFFECTS}

Perturbations in the threshold voltages will change the output of the digital correlator. With asymmetric threshold levels, the 
digital correlation coefficient can be computed by the following:

$$
\begin{aligned}
r_{a b}= & \left.r_{a b}\right|_{\rho=0} \\
& +\sigma_{v_{a}} \sigma_{v_{b}} \int_{0}^{\rho} f\left(\sigma_{v_{a}}\left(\theta_{a}+\delta_{a}\right), \sigma_{v_{b}}\left(\theta_{b}+\delta_{b}\right), \rho^{\prime}\right) \\
& +f\left(\sigma_{v_{a}}\left(\theta_{a}+\delta_{a}\right), \sigma_{v_{b}}\left(-\theta_{b}+\delta_{b}\right), \rho^{\prime}\right) \\
& +f\left(\sigma_{v_{a}}\left(-\theta_{a}+\delta_{a}\right), \sigma_{v_{b}}\left(\theta_{b}+\delta_{b}\right), \rho^{\prime}\right) \\
& +f\left(\sigma_{v_{a}}\left(-\theta_{a}+\delta_{a}\right), \sigma_{v_{b}}\left(-\theta_{b}+\delta_{b}\right), \rho^{\prime}\right) d \rho^{\prime} .
\end{aligned}
$$

Using the definition for the bivariate normal pdf (73), the previous equation becomes

$$
\begin{aligned}
r_{a b}= & \left.r_{a b}\right|_{\rho=0}+\int_{0}^{\rho} p\left(\theta_{a}+\delta_{a}, \theta_{b}+\delta_{b}, \rho^{\prime}\right) \\
& +p\left(\theta_{a}+\delta_{a},-\theta_{b}+\delta_{b}, \rho^{\prime}\right) \\
& +p\left(-\theta_{a}+\delta_{a}, \theta_{b}+\delta_{b}, \rho^{\prime}\right) \\
& +p\left(-\theta_{a}+\delta_{a},-\theta_{b}+\delta_{b}, \rho^{\prime}\right) d \rho^{\prime} .
\end{aligned}
$$

To determine the behavior of $r_{a b}$ with respect to $\delta_{a}$ and $\delta_{b}$, we need to express the integrand in a 3-D power series in $\rho, \delta_{a}$ and $\delta_{b}$. The integrand is

$$
\begin{aligned}
I\left(\rho^{\prime}\right)= & p\left(\theta_{a}+\delta_{a}, \theta_{b}+\delta_{b}, \rho^{\prime}\right)+p\left(\theta_{a}+\delta_{a},-\theta_{b}+\delta_{b}, \rho^{\prime}\right) \\
& +p\left(-\theta_{a}+\delta_{a}, \theta_{b}+\delta_{b}, \rho^{\prime}\right) \\
& +p\left(-\theta_{a}+\delta_{a},-\theta_{b}+\delta_{b}, \rho^{\prime}\right)
\end{aligned}
$$

and its expansion in $\rho^{\prime}$ is nearly identical to that in Appendix I. The Taylor series expansion is

$$
\begin{array}{r}
I\left(\rho^{\prime}\right)=I(0)+I^{(1)}(0) \rho^{\prime}+\frac{1}{2 !} I^{(2)}(0) \rho^{2}+\frac{1}{3 !} I^{(3)}(0) \rho^{\prime 3} \\
+\frac{1}{4 !} I^{(4)}(0) \rho^{4}+\cdots
\end{array}
$$

What remains is to determine the two-dimensional (2-D) Taylor series in $\delta_{a}$ and $\delta_{b}$ of each of the terms of the integrand. Throughout, it is assumed that $\delta_{a}$ and $\delta_{b}$ are $\mathcal{O}(\delta)$, and the Taylor series are generally truncated beyond $\mathcal{O}\left(\delta^{2}\right)$.

The first term of $I\left(\rho^{\prime}\right)$ is

$$
\begin{aligned}
I(0)= & p\left(\theta_{a}+\delta_{a}, \theta_{b}+\delta_{b}, 0\right)+p\left(\theta_{a}+\delta_{a},-\theta_{b}+\delta_{b}, 0\right) \\
& +p\left(-\theta_{a}+\delta_{a}, \theta_{b}+\delta_{b}, 0\right)+p\left(-\theta_{a}+\delta_{a},-\theta_{b}+\delta_{b}, 0\right) .
\end{aligned}
$$

There are four instances of a term with the form $p(x+a, y+b, 0)$, where $x= \pm \theta_{a}, y= \pm \theta_{b}, a=\delta_{a}, b=\delta_{b}$. The 2-D Taylor series about $x$ and $y$ will be applied to the previous equation.

The expression $p(x+a, y+b, 0)$ is the product of two standard normal pdfs: $p(x+a, y+b, 0)=Z(x+a) Z(y+b)$, where $Z(x)=1 /(\sqrt{2 \pi}) e^{-x^{2} / 2}$ is the standard normal curve ([21 (26.2.1)]. The Taylor series of this product is

$$
\begin{aligned}
p(x+ & a, y+b, 0) \\
= & Z(x) Z(y)+Z^{(1)}(x) Z(y) a \\
& +Z(x) Z^{(1)}(y) b+\frac{1}{2 !}\left[Z^{(2)}(x) Z(y) a^{2}\right. \\
& \left.+2 Z^{(1)}(x) Z^{(1)}(y) a b+Z(x) Z^{(2)}(y) b^{2}\right]+\cdots .
\end{aligned}
$$

The derivatives $Z^{(n)}(x)$ of the standard normal pdf can be expressed in terms of Hermite polynomials ([21 (26.2.32)]

$$
Z^{(n)}(x)=(-1)^{-n} 2^{-n / 2} Z(x) H_{n}\left(\frac{x}{\sqrt{2}}\right)
$$

where the first three Hermite polynomials are $H_{0}(x)=$ $1, H_{1}(x)=2 x$, and $H_{2}(x)=4 x^{2}-2$. For example, the second derivative of $Z(x)$ is

$$
\begin{aligned}
Z^{(2)}(x) & =(-1)^{-2} 2^{-2 / 2} Z(x) H_{2}\left(\frac{x}{\sqrt{2}}\right) \\
& =Z(x)\left(x^{2}-1\right) .
\end{aligned}
$$

Substituting these results into (116), the first term of the integrand is

$$
\begin{aligned}
I(0)= & Z\left(\theta_{a}\right) Z\left(\theta_{b}\right)+Z^{(1)}\left(\theta_{a}\right) Z\left(\theta_{b}\right) \delta_{a} \\
& +Z\left(\theta_{a}\right) Z^{(1)}\left(\theta_{b}\right) \delta_{b}+\frac{1}{2 !}\left[Z^{(2)}\left(\theta_{a}\right) Z\left(\theta_{b}\right) \delta_{a}^{2}\right. \\
& \left.+2 Z^{(1)}\left(\theta_{a}\right) Z^{(1)}\left(\theta_{b}\right) \delta_{a} \delta_{b}+Z\left(\theta_{a}\right) Z^{(2)}\left(\theta_{b}\right) \delta_{b}^{2}\right] \\
& +Z\left(\theta_{a}\right) Z\left(\theta_{b}\right)+Z^{(1)}\left(\theta_{a}\right) Z\left(\theta_{b}\right) \delta_{a} \\
& -Z\left(\theta_{a}\right) Z^{(1)}\left(\theta_{b}\right) \delta_{b}+\frac{1}{2 !}\left[Z^{(2)}\left(\theta_{a}\right) Z\left(\theta_{b}\right) \delta_{a}^{2}\right. \\
& \left.-2 Z^{(1)}\left(\theta_{a}\right) Z^{(1)}\left(\theta_{b}\right) \delta_{a} \delta_{b}+Z\left(\theta_{a}\right) Z^{(2)}\left(\theta_{b}\right) \delta_{b}^{2}\right] \\
& +Z\left(\theta_{a}\right) Z\left(\theta_{b}\right)-Z^{(1)}\left(\theta_{a}\right) Z\left(\theta_{b}\right) \delta_{a} \\
& +Z\left(\theta_{a}\right) Z^{(1)}\left(\theta_{b}\right) \delta_{b}+\frac{1}{2 !}\left[Z^{(2)}\left(\theta_{a}\right) Z\left(\theta_{b}\right) \delta_{a}^{2}\right. \\
& \left.-2 Z^{(1)}\left(\theta_{a}\right) Z^{(1)}\left(\theta_{b}\right) \delta_{a} \delta_{b}+Z\left(\theta_{a}\right) Z^{(2)}\left(\theta_{b}\right) \delta_{b}^{2}\right] \\
& +Z\left(\theta_{a}\right) Z\left(\theta_{b}\right)-Z^{(1)}\left(\theta_{a}\right) Z\left(\theta_{b}\right) \delta_{a} \\
& -Z\left(\theta_{a}\right) Z^{(1)}\left(\theta_{b}\right) \delta_{b}+\frac{1}{2 !}\left[Z^{(2)}\left(\theta_{a}\right) Z\left(\theta_{b}\right) \delta_{a}^{2}\right. \\
& \left.+2 Z^{(1)}\left(\theta_{a}\right) Z^{(1)}\left(\theta_{b}\right) \delta_{a} \delta_{b}+Z\left(\theta_{a}\right) Z^{(2)}\left(\theta_{b}\right) \delta_{b}^{2}\right]+\cdots \\
= & 4 p(x, y, 0)\left(1-\frac{1}{2}\left[\left(1-\theta_{a}^{2}\right) \delta_{a}^{2}+\left(1-\theta_{b}^{2}\right) \delta_{b}^{2}\right]\right) \\
& +\mathcal{O}\left(\delta^{3}\right) .
\end{aligned}
$$

The second term in the Taylor series of $I\left(\rho^{\prime}\right)$ is

$$
\begin{aligned}
I^{(1)}(0) \rho^{\prime}= & {\left[\left.\frac{\partial}{\partial \rho} p\left(\theta_{a}+\delta_{a}, \theta_{b}+\delta_{b}, \rho\right)\right|_{\rho=0}\right.} \\
& +\left.\frac{\partial}{\partial \rho} p\left(\theta_{a}+\delta_{a},-\theta_{b}+\delta_{b}, \rho\right)\right|_{\rho=0} \\
& +\left.\frac{\partial}{\partial \rho} p\left(-\theta_{a}+\delta_{a}, \theta_{b}+\delta_{b}, \rho\right)\right|_{\rho=0} \\
& \left.+\left.\frac{\partial}{\partial \rho} p\left(-\theta_{a}+\delta_{a},-\theta_{b}+\delta_{b}, \rho\right)\right|_{\rho=0}\right] \rho^{\prime}
\end{aligned}
$$

The first partial derivative with respect to $\rho$, evaluated at zero, of the bivariate normal pdf is

$$
\begin{aligned}
\left.\frac{\partial}{\partial \rho} p\left(\theta_{a}, \theta_{b}, \rho\right)\right|_{\rho=0} & =p\left(\theta_{a}, \theta_{b}, 0\right) \theta_{a} \theta_{b} \\
& =\theta_{a} Z\left(\theta_{a}\right) \theta_{b} Z\left(\theta_{b}\right) .
\end{aligned}
$$


Thus

$$
\begin{aligned}
& I^{(1)}(0) \\
&=\left(\theta_{a}+\delta_{a}\right) Z\left(\theta_{a}+\delta_{a}\right)\left(\theta_{b}+\delta_{b}\right) Z\left(\theta_{b}+\delta_{b}\right) \\
&+\left(\theta_{a}+\delta_{a}\right) Z\left(\theta_{a}+\delta_{a}\right)\left(-\theta_{b}+\delta_{b}\right) Z\left(-\theta_{b}+\delta_{b}\right) \\
&+\left(-\theta_{a}+\delta_{a}\right) Z\left(-\theta_{a}+\delta_{a}\right)\left(\theta_{b}+\delta_{b}\right) Z\left(\theta_{b}+\delta_{b}\right) \\
&+\left(-\theta_{a}+\delta_{a}\right) Z\left(-\theta_{a}+\delta_{a}\right)\left(-\theta_{b}+\delta_{b}\right) Z\left(-\theta_{b}+\delta_{b}\right) \\
&= {\left[\left(\theta_{a}+\delta_{a}\right) Z\left(\theta_{a}+\delta_{a}\right)+\left(-\theta_{a}+\delta_{a}\right) Z\left(-\theta_{a}+\delta_{a}\right)\right] } \\
& \times\left[\left(\theta_{b}+\delta_{b}\right) Z\left(\theta_{b}+\delta_{b}\right)+\left(-\theta_{b}+\delta_{b}\right) Z\left(-\theta_{b}+\delta_{b}\right)\right] .
\end{aligned}
$$

Next, consider the expression $\left(\theta_{\alpha}+\delta_{\alpha}\right) Z\left(\theta_{\alpha}+\delta_{\alpha}\right)+\left(-\theta_{\alpha}+\right.$ $\left.\delta_{\alpha}\right) Z\left(-\theta_{\alpha}+\delta_{\alpha}\right)$ by expanding the standard normal pdfs in 1-D Taylor series

$$
\begin{aligned}
\left(\theta_{\alpha}+\right. & \left.\delta_{\alpha}\right)\left[Z\left(\theta_{\alpha}\right)+Z^{(1)}\left(\theta_{\alpha}\right) \delta_{\alpha}+\frac{1}{2 !} Z^{(2)}\left(\theta_{\alpha}\right) \delta_{\alpha}+\cdots\right] \\
+ & \left(-\theta_{\alpha}+\delta_{\alpha}\right)\left[Z\left(-\theta_{\alpha}\right)+Z^{(1)}\left(-\theta_{\alpha}\right) \delta_{\alpha}\right. \\
+ & \left.\frac{1}{2 !} Z^{(2)}\left(-\theta_{\alpha}\right) \delta_{\alpha}^{2}+\cdots\right] \\
= & \theta_{\alpha}\left[Z\left(\theta_{\alpha}\right)+Z^{(1)}\left(\theta_{\alpha}\right) \delta_{\alpha}+\frac{1}{2 !} Z^{(2)}\left(\theta_{\alpha}\right) \delta_{\alpha}^{2}+\cdots\right] \\
& -\theta_{\alpha}\left[Z\left(\theta_{\alpha}\right)-Z^{(1)}\left(\theta_{\alpha}\right) \delta_{\alpha}+\frac{1}{2 !} Z^{(2)}\left(\theta_{\alpha}\right) \delta_{\alpha}^{2}+\cdots\right] \\
& +\delta_{\alpha}\left[Z\left(\theta_{\alpha}\right)+Z^{(1)}\left(\theta_{\alpha}\right) \delta_{\alpha}+\frac{1}{2 !} Z^{(2)}\left(\theta_{\alpha}\right) \delta_{\alpha}^{2}+\cdots\right] \\
& +\delta_{\alpha}\left[Z\left(\theta_{\alpha}\right)-Z^{(1)}\left(\theta_{\alpha}\right) \delta_{\alpha}+\frac{1}{2 !} Z^{(2)}\left(\theta_{\alpha}\right) \delta_{\alpha}^{2}+\cdots\right] \\
= & \theta_{\alpha}\left(2 Z^{(1)}\left(\theta_{\alpha}\right) \delta_{\alpha}+\cdots\right) \\
& +2 \delta_{\alpha}\left[Z\left(\theta_{\alpha}\right)+\frac{1}{2 !} Z^{(2)}\left(\theta_{\alpha}\right) \delta_{\alpha}^{2}+\cdots\right] \\
= & Z\left(\theta_{\alpha}\right) \delta_{\alpha}\left(1-2 \theta_{\alpha}^{2}\right)+\mathcal{O}\left(\delta_{\alpha}^{3}\right) .
\end{aligned}
$$

Finally, substituting the previous series into the $a$ and $b$ terms of (123) yields the following:

$$
I^{(1)}(0)=2 \delta_{a} \delta_{b} p\left(\theta_{a}, \theta_{b}, 0\right)\left(1-2 \theta_{a}^{2}\right)\left(1-2 \theta_{b}^{2}\right)+\mathcal{O}\left(\delta^{3}\right) .
$$

The third term in the series expansion of $I\left(\rho^{\prime}\right)$ contains

$$
\begin{aligned}
I^{(2)}(0)= & {\left[\left(\theta_{a}+\delta_{a}\right)^{2}-1\right] Z\left(\theta_{a}+\delta_{a}\right)\left[\left(\theta_{b}+\delta_{b}\right)^{2}-1\right] } \\
& \times Z\left(\theta_{b}+\delta_{b}\right)+\left[\left(\theta_{a}+\delta_{a}\right)^{2}-1\right] Z\left(\theta_{a}+\delta_{a}\right) \\
& \times\left[\left(-\theta_{b}+\delta_{b}\right)^{2}-1\right] Z\left(-\theta_{b}+\delta_{b}\right) \\
& +\left[\left(-\theta_{a}+\delta_{a}\right)^{2}-1\right] Z\left(-\theta_{a}+\delta_{a}\right) \\
& \times\left[\left(\theta_{b}+\delta_{b}\right)^{2}-1\right] Z\left(\theta_{b}+\delta_{b}\right) \\
& +\left[\left(-\theta_{a}+\delta_{a}\right)^{2}-1\right] Z\left(-\theta_{a}+\delta_{a}\right) \\
& \times\left[\left(-\theta_{b}+\delta_{b}\right)^{2}-1\right] Z\left(-\theta_{b}+\delta_{b}\right) \\
= & {\left[\left(\theta_{a}^{2}+2 \theta_{a} \delta_{a}+\delta_{a}^{2}-1\right) Z\left(\theta_{a}+\delta_{a}\right)\right.} \\
& \left.+\left(\theta_{a}^{2}-2 \theta_{a} \delta_{a}+\delta_{a}^{2}-1\right) Z\left(-\theta_{a}+\delta_{a}\right)\right] \\
& \times\left[\left(\theta_{b}^{2}+2 \theta_{b} \delta_{b}+\delta_{b}^{2}-1\right) Z\left(\theta_{b}+\delta_{b}\right)\right. \\
& \left.+\left(\theta_{b}^{2}-2 \theta_{b} \delta_{b}+\delta_{b}^{2}-1\right) Z\left(-\theta_{b}+\delta_{b}\right)\right] . \quad(126)
\end{aligned}
$$

Consider the following expression by expanding $Z$ in a power series:

$$
\begin{aligned}
& \left(\theta_{\alpha}^{2}+2 \theta_{\alpha} \delta_{\alpha}+\delta_{\alpha}^{2}-1\right) Z\left(\theta_{\alpha}+\delta_{\alpha}\right) \\
& \quad+\left(\theta_{\alpha}^{2}-2 \theta_{\alpha} \delta_{\alpha}+\delta_{\alpha}^{2}-1\right) Z\left(-\theta_{\alpha}+\delta_{\alpha}\right)
\end{aligned}
$$

$$
\begin{aligned}
= & \left(\theta_{\alpha}^{2}+2 \theta_{\alpha} \delta_{\alpha}+\delta_{\alpha}^{2}-1\right)\left[Z\left(\theta_{\alpha}\right)+Z^{(1)}\left(\theta_{\alpha}\right) \delta_{\alpha}\right. \\
& \left.+\frac{1}{2 !} Z^{(2)}\left(\theta_{\alpha}\right) \delta_{\alpha}^{2}+\cdots\right]+\left(\theta_{\alpha}^{2}-2 \theta_{\alpha} \delta_{\alpha}+\delta_{\alpha}^{2}-1\right) \\
& \times\left[Z\left(-\theta_{\alpha}\right)+Z^{(1)}\left(-\theta_{\alpha}\right) \delta_{\alpha}\right. \\
& \left.+\frac{1}{2 !} Z^{(2)}\left(-\theta_{\alpha}\right) \delta_{\alpha}^{2}+\cdots\right] \\
= & 2\left(\theta_{\alpha}^{2}+\delta_{\alpha}^{2}-1\right)\left[Z\left(\theta_{\alpha}\right)+\frac{1}{2 !} Z^{(2)}\left(\theta_{\alpha}\right) \delta_{\alpha}^{2}+\cdots\right] \\
& +4 \theta_{\alpha} \delta_{\alpha}\left[Z^{(1)}\left(\theta_{\alpha}\right) \delta_{\alpha}+\cdots\right] \\
= & 2\left(\theta_{\alpha}^{2}+\delta_{\alpha}^{2}-1\right)\left[Z\left(\theta_{\alpha}\right)+\frac{1}{2 !} Z\left(\theta_{\alpha}\right)\left(\theta_{\alpha}^{2}-1\right) \delta_{\alpha}^{2}\right] \\
& +8 \theta_{\alpha} \delta_{\alpha} \theta_{\alpha} Z\left(\theta_{\alpha}\right) \delta_{\alpha}+\cdots \\
= & Z\left(\theta_{\alpha}\right)\left(\left(\theta_{\alpha}^{2}-1\right) \delta_{\alpha}^{4}\right. \\
& \left.+\left(6 \theta_{\alpha}^{2}+\theta_{\alpha}^{4}+3\right) \delta_{\alpha}^{2}+2 \theta_{\alpha}^{2}-2\right)+\cdots
\end{aligned}
$$

Substituting the previous result into (126) produces $I^{(2)}(0)=p\left(\theta_{a}, \theta_{b}, 0\right)\left[\left(6 \theta_{a}^{2}+\theta_{a}^{4}+3\right) \delta_{a}^{2}+2 \theta_{a}^{2}-2\right]$

$$
\times\left[\left(6 \theta_{b}^{2}+\theta_{b}^{4}+3\right) \delta_{b}^{2}+2 \theta_{b}^{2}-2\right]+\mathcal{O}\left(\delta^{4}\right) .
$$

Combining and integrating the first three terms of the integrand (120), (125), and (128) results in

$$
\begin{aligned}
r_{a b}= & \left.r_{a b}\right|_{\rho=0}+4 p\left(\theta_{a}, \theta_{b}, 0\right) \\
& \times\left(1-\frac{1}{2}\left[\left(1-\theta_{a}^{2}\right) \delta_{a}^{2}+\left(1-\theta_{b}^{2}\right) \delta_{b}^{2}\right]\right) \rho \\
& +\delta_{a} \delta_{b} p\left(\theta_{a}, \theta_{b}, 0\right)\left(1-2 \theta_{a}^{2}\right)\left(1-2 \theta_{b}^{2}\right) \rho^{2} \\
& +\frac{1}{3} p\left(\theta_{a}, \theta_{b}, 0\right)\left(\left(6 \theta_{a}^{2}+\theta_{a}^{4}+3\right) \delta_{a}^{2}+2 \theta_{a}^{2}-2\right) \\
& \times\left(\left(6 \theta_{b}^{2}+\theta_{b}^{4}+3\right) \delta_{b}^{2}+2 \theta_{b}^{2}-2\right) \rho^{3}+\mathcal{O}\left(\rho^{4}, \delta^{3}\right) \\
= & \left.r_{a b}\right|_{\rho=0}+4 p\left(\theta_{a}, \theta_{b}, 0\right)\left[\rho+\frac{1}{6}\left(\theta_{a}^{2}-1\right)\left(\theta_{b}^{2}-1\right) \rho^{3}\right] \\
& +4 p\left(\theta_{a}, \theta_{b}, 0\right)\left[-\frac{1}{2}\left[\left(1-\theta_{a}^{2}\right) \delta_{a}^{2}+\left(1-\theta_{b}^{2}\right) \delta_{b}^{2}\right] \rho\right. \\
& +\frac{1}{4} \delta_{a} \delta_{b}\left(1-2 \theta_{a}^{2}\right)\left(1-2 \theta_{b}^{2}\right) \rho^{2} \\
& -\frac{1}{6}\left[\left(6 \theta_{a}^{2}+\theta_{a}^{4}+3\right)\left(1-\theta_{b}^{2}\right) \delta_{a}^{2}\right. \\
& \left.\left.+\left(1-\theta_{a}^{2}\right)\left(6 \theta_{b}^{2}+\theta_{b}^{4}+3\right) \delta_{b}^{2}\right] \rho^{3}\right]+\mathcal{O}\left(\rho^{4}, \delta^{3}\right) .
\end{aligned}
$$

There are two different power series in $\rho$ that can be identified in the previous equation. These are the ideal relationship between $\rho$ and $r$ and an error series caused by nonzero threshold offsets $\delta_{a}$ and $\delta_{b}$

$$
\begin{aligned}
r_{a b}= & \left.r_{a b}\right|_{\rho=0}+\left.r_{a b}\right|_{a}=\delta_{b}=0+\frac{2}{\pi} \exp \left[-\frac{1}{2}\left(\theta_{a}^{2}+\theta_{b}^{2}\right)\right] \\
& \times\left[-\frac{1}{2}\left[\left(1-\theta_{a}^{2}\right) \delta_{a}^{2}+\left(1-\theta_{b}^{2}\right) \delta_{b}^{2}\right] \rho\right. \\
& +\frac{1}{4} \delta_{a} \delta_{b}\left(1-2 \theta_{a}^{2}\right)\left(1-2 \theta_{b}^{2}\right) \rho^{2} \\
& -\frac{1}{6}\left[\left(6 \theta_{a}^{2}+\theta_{a}^{4}+3\right)\left(1-\theta_{b}^{2}\right) \delta_{a}^{2}+\left(1-\theta_{a}^{2}\right)\right. \\
& \left.\left.\times\left(6 \theta_{b}^{2}+\theta_{b}^{4}+3\right) \delta_{b}^{2}\right] \rho^{3}\right]+\mathcal{O}\left(\rho^{4}, \delta^{3}\right) .
\end{aligned}
$$




\section{ACKNOWLEDGMENT}

The authors would like to thank W. Blackwell and M. Klein for the help in designing and fabricating the PSR/D correlators, and D. Kunkee and P. Lowe for their help on the polarized calibration load demonstration. They would also like to thank Drs. R. Kakar, S. Mango, and M. Van Woert for their support of this work.

\section{REFERENCES}

[1] J. R. Piepmeier and A. J. Gasiewski, "High-resolution passive polarimetric microwave mapping of ocean surface wind vector fields," IEEE Trans. Geosci. Remote Sensing, vol. 39, pp. 606-622, May 2001.

[2] P. W. Rosenkranz and D. H. Staelin, "Polarized thermal microwave emission from oxygen in the mesosphere," Radio Sci., vol. 23, no. 5, pp. 721-729, 1988

[3] L. Tsang, J. A. Kong, and R. T. Shin, Theory of Microwave Remote Sensing. New York: Wiley, 1985.

[4] F. T. Ulaby, R. K. Moore, and A. K. Fung, Microwave Remote Sensing: Active and Passive. Reading, MA: Addison-Wesley, 1986.

[5] S. Chandrasekhar, Radiative Transfer. New York: Dover, 1960

[6] H. Stark and J. W. Woods, Probability, Random Processes, and Estimation Theory for Engineers, 2nd ed. Englewood Cliffs, NJ: Prentice-Hall, 1994.

[7] M. A. Fischman and A. W. England, "Sensitivity of a $1.4 \mathrm{GHz}$ directsampling digital radiometer," IEEE Trans. Geosci. Remote Sensing, vol. 37, pp. 2172-2180, Sept. 1999

[8] A. J. Gasiewski and D. B. Kunkee, "Calibration and applications of polarization-correlating radiometers," IEEE Trans. Microwave Theory Tech., vol. 41, May 1993.

[9] J. Lahtinen, A. J. Gasiewski, M. Klein, V. Leuisky, and A. Yevgrofov, "Fully Polarized Microwave Radiometer Calibration," IEEE Trans. Geosci. Remote Sensing, to be published.

[10] S. Weinreb, "Digital radiometer," Proc. IEEE, vol. 49, p. 1099, June 1961.

[11] B. F. C. Cooper, "Correlators with two-bit quantization," Aust. J. Phys., vol. 23, pp. 521-527, 1970.

[12] S. Padin et al., "High-speed digital correlator for radio astronomy," IEEE Trans. Instr. Measure., vol. 42, no. 4, pp. 793-798, 1993.

[13] J. Canaris, "NASA CERC digital correlator projects," in 4th NASA Symp. VLSI Design. Moscow, ID, 1992

[14] D. W. Hawkins and D. P. Woody, "A $500 \mathrm{MHz}$ bandwidth, 512 lag, digital cross correlator," in Proc. Nat. Radio Science Meeting, Boulder, CO, 1998, p. 160.

[15] C. Timoc and T. Tran, "Digital correlation spectrometer with $1 \mathrm{GHz}$ sampling rate," in Proc. Nat. Radio Science Meeting, Boulder, CO, 1998, p. 161.

[16] S. K. Okumura, M. Momose, T. Tsutsumi, and N. Kawaguchi, "1-GHz bandwidth digital spetro-correlator system for Nobeyama millimeter array," in Proc. Nat. Radio Science Meeting, Boulder, CO, 1998, p. 162.

[17] R. Price, "A useful theorem for nonlinear devices having Gaussian inputs," IRE Trans. Inform. Theory, vol. IT-4, pp. 69-72, Jan. 1958.

[18] J. B. Hagen and D. T. Farley, "Digital-correlation techniques in radio science," Radio Sci., vol. 8, no. 8-9, pp. 775-784, 1973.

[19] L. R. D' Addario et al., "Complex cross correlators with three-level quantization: Design tolerances," Radio Sci., vol. 19, no. 3, pp. 931-945, 1984.

[20] S. R. Kulkarni and C. Heiles, "How to obtain the true correlation from a 3-level digital correlator," Astronomy J., vol. 85, no. 10, pp. 1413-1420, 1980.

[21] Handbook of Mathematical Functions, Dover, New York, 1964.

[22] A. R. Thompson, J. M. Morgan, and G. W. Swenson Jr., Interferometry and Synthesis in Radio Astronomy. Malabar, FL: Krieger, 1991.

[23] J. R. Piepmeier and A. J. Gasiewski, "Polarimetric scanning radiometer for airborne microwave imaging studies," in Proc. IGARSS'96. Lincoln, NE, 1996, pp. 1688-1691.

[24] — , "High-resolution multiband passive polarimetric observations of the ocean surface," in Proc. IGARSS'97, vol. 2, Singapore, 1997, pp. $1006-1008$

[25] J. R. Piepmeier, A. J. Gasiewski, M. Klein, V. Boehm, and R. C. Lum, "Ocean surface wind direction measurement by scanning polarimetric microwave radiometry," in Proc. IGARSS'98, vol. 5, Seattle, WA, 1998, pp. $2307-2310$
[26] D. B. Kunkee and A. J. Gasiewski, "Simulation of passive microwave wind direction signatures over the ocean using an asymmetric-wave geometrical optics model," Radio Sci., vol. 32, no. 1, pp. 59-77, 1997.

[27] S. H. Yueh, "Modeling of wind direction signals in polarimetric sea surface brightness temperatures," IEEE Trans. Geosci. Remote Sensing, vol. 35, pp. 1400-1418, Nov. 1997.

[28] F. J. Wentz, "Measurement of oceanic wind vector using satellite microwave radiometers," IEEE Trans. Geosci. Remote Sensing, vol. 30, pp. 960-972, Sept. 1992

[29] M. S. Dzura et al., "Radiometers-polarimeters: Principles of design and applications for sea surface microwave emission polarimetry," in Proc. IGARSS'92, Houston, TX, 1992.

[30] A. J. Gasiewski et al., "Combined high-resolution active and passive imaging of ocean surface winds from aircraft," in Proc. IGARSS' 97, Singapore, 1997, pp. 1001-1005.

[31] S. Yueh, W. Wilson, F. Li, W. Ricketts, and S. Nghiem, "Polarimetric brightness temperature of sea surfaces measured with K- and Ka-band radiometers," IEEE Trans. Geosci. Remote Sensing, vol. 35, pp. 1177-1187, Sept. 1997.

[32] D. M. Le Vine, "Synthetic aperture radiometer systems," IEEE Trans. Geosci. Remote Sensing, vol. 47, pp. 2228-2236, Nov. 1999.

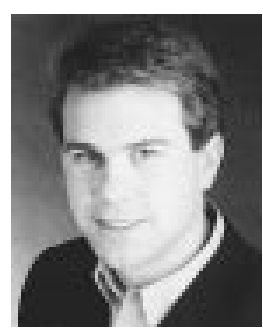

Jeffrey R. Piepmeier (M'99) received the B.S. degree in engineering from LeTourneau University, Longview, TX, in 1993, the M.S. degree in electrical engineering from Georgia Institute of Technology (Georgia Tech), Atlanta, in 1994, and the Ph.D. degree in electrical engineering from Georgia Tech in 1999.

From 1993 to 1994, he was a Schakleford Fellow with the Georgia Tech Research Institute. He is currently with the Microwave Instrument Technology Branch of NASA's Goddard Space Flight Center, Greenbelt, MD. His technical interests include passive remote sensing, microwave radiometers and polarimetry, and microwave digital circuits.

Dr. Piepmeier was Conference Chair of the 2000 Microwave Radiometer Calibration Workshop. He is a member of URSI (Commission F) and the American Geophysical Union.

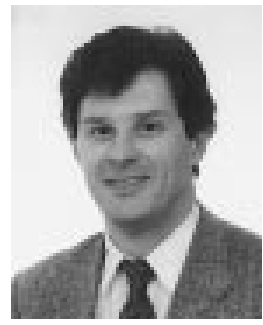

Albin J. Gasiewski (M'88-SM'95) received the B.S. and M.S. degrees in electrical engineering and the B.S. degree in mathematics, all from Case Western Reserve University, Cleveland, OH, in 1983, and the Ph.D. degree in electrical engineering and computer science from the Massachusetts Institute of Technology, Cambridge, in 1989.

From 1989 to 1997, he was a Faculty Member within the School of Electrical and Computer Engineering, Georgia Institute of Technology (Georgia Tech), Atlanta. He was an Associate Professor at Georgia Tech, where he taught courses on electromagnetics, remote sensing, instrumentation, and wave propagation theory. He is currently with the NOAA Environmental Technology Laboratory, Boulder, CO, where he is Acting Chief of the ETL Microwave Systems Development Division. His technical interests include passive and active remote sensing, radiative transfer theory, electromagnetics, antennas and microwave circuits, electronic instrumentation, meteorology, and oceanography.

Dr. Gasiewski is a member of the IEEE Geoscience and Remote Sensing Society's AdCom, was General Chair of the second Combined Optical-Microwave Earth and Atmosphere Sensing Symposium (1995), and Technical Program Committee Chair of the 20th International Geoscience and Remote Sensing Symposium (IGARSS 2000). He is a member of Eta Kappa Nu, Tau Beta Pi, URSI (Commission F), Sigma Xi, the American Meteorological Society, COSPAR, and the American Geophysical Union. He served on the U.S. National Research Council's Committee on Radio Frequencies (CORF) from 1989 to 1995 and is the author or co-author of 52 publications in the area of electromagnetic remote sensing. 